QUARTERLY OF APPLIED MATHEMATICS

VOLUME LXX, NUMBER 4

DECEMBER 2012, PAGES 721-742

S 0033-569X(2012)01269-3

Article electronically published on June 21, 2012

\title{
LONG TIME BEHAVIOR OF THE FOKKER-PLANCK-BOLTZMANN EQUATION WITH SOFT POTENTIAL
}

\author{
BY \\ MING-YING ZHONG (Department of Mathematical Sciences, Tsinghua University, Beijing, 100804 \\ People's Republic of China) \\ AND \\ HAI-LIANG LI (Department of Mathematics, Capital Normal University, Beijing, 100037 People's \\ Republic of China)
}

Abstract. In the present paper, we consider the initial value problem for the FokkerPlanck-Boltzmann equation with soft potential. For initial data near an absolute Maxwellian, we show the global existence and uniqueness of the classical solution and establish its long time decay rate.

\section{Introduction and main result}

When an equation is concerned with the motion of particles in a thermal bath for which the bilinear interaction is one of the main characters, we have the Fokker-PlanckBoltzmann type equation. The equations of such type have also been used recently in the description of grazing collisions [4, in the area of aerosols [26] and in driven media 2. In the present paper, we consider the initial value problem (IVP) of the FokkerPlanck-Boltzmann (FPB) equation

$$
\begin{gathered}
F_{t}+v \cdot \nabla_{x} F=\mathcal{Q}(F, F)+\varepsilon L_{F P} F, \\
F(x, v, t)=F_{0}(x, v),
\end{gathered}
$$

where $F=F(x, v, t),(x, v, t) \in \mathbb{R}^{3} \times \mathbb{R}^{3} \times \mathbb{R}^{+}$, is the distribution function and $\varepsilon>0$ is a given constant. The Fokker-Planck operator $L_{F P}$ is defined by

$$
L_{F P} F=\nabla_{v} \cdot\left(\nabla_{v} F+v F\right) .
$$

Received November 27, 2010.

2010 Mathematics Subject Classification. Primary 82C40, 35Q20, 35Q84.

The research for this paper was partially supported by the NNSFC Nos. 10871134, 10910257 and 11011130029, the Huo Ying Dong Fund No. 111033, and the AHRDIHL Project of Beijing Municipality No. PHR201006107.

E-mail address: zhongmingying@sina.com

E-mail address: hailiang.li.math@gmail.com

(C) 2012 Brown University Reverts to public domain 28 years from publication 
The bilinear collision operator $\mathcal{Q}(F, G)$ is given by (see [30])

$$
\mathcal{Q}(F, G)=\int_{\mathbb{R}^{3}} \int_{\mathbb{S}^{2}}|u-v|^{\gamma}\left(F\left(u^{\prime}\right) G\left(v^{\prime}\right)-F(u) G(v)\right) B(\theta) d u d \omega,
$$

where

$$
u^{\prime}=u-[(u-v) \cdot \omega] \omega, \quad v^{\prime}=v+[(u-v) \cdot \omega] \omega, \quad \omega \in \mathbb{S}^{2} .
$$

In the present paper, we consider the case of soft potential $-1 \leq \gamma<0$ and assume that $B(\theta)$ satisfies Grad's angular cutoff assumption

$$
0<B(\theta) \leq C|\cos \theta|,
$$

where $C>0$ is a constant.

Denote a normalized global Maxwellian by

$$
\mu(v)=e^{-\frac{|v|^{2}}{2}},
$$

and set $f(x, v, t)$ to be the perturbation of $F$ near $\mu$

$$
F=\mu+\sqrt{\mu} f .
$$

Then, from IVP (1.1)-(1.2), the corresponding initial value problem for the perturbation $f$ takes

$$
\begin{gathered}
f_{t}+v \cdot \nabla_{x} f=\mathbf{L} f+\Gamma(f, f)+\varepsilon \mathbf{F} f \\
f(x, v, 0)=f_{0}(x, v)=:\left(F_{0}-\mu\right) \mu^{-\frac{1}{2}}
\end{gathered}
$$

where

$$
\begin{aligned}
\mathbf{L} f & =\mu^{-\frac{1}{2}}\left(\mathcal{Q}\left(\mu, \mu^{\frac{1}{2}} f\right)+\mathcal{Q}\left(\mu^{\frac{1}{2}} f, \mu\right)\right), \\
\Gamma(f, f) & =\mu^{-\frac{1}{2}} \mathcal{Q}\left(\mu^{\frac{1}{2}} f, \mu^{\frac{1}{2}} f\right), \\
\mathbf{F} f & =\mu^{-\frac{1}{2}} \nabla_{v} \cdot\left(\nabla_{v}\left(\mu^{\frac{1}{2}} f\right)+v \mu^{\frac{1}{2}} f\right) .
\end{aligned}
$$

The linear operator $\mathbf{L}$ is defined by (see [1])

$$
\mathbf{L} g=\nu(v) g-K g,
$$

where $\nu(v)$ is the collision frequency given by

$$
\nu(v)=\int|u-v|^{\gamma} \mu(u) B(\theta) d u d \omega
$$

satisfying $\nu_{0}(1+|v|)^{\gamma} \leq \nu(v) \leq \nu_{1}(1+|v|)^{\gamma}$ for some constants $\nu_{0}, \nu_{1}>0$, and $K$ is the compact operator in $L^{2}\left(\mathbb{R}^{3}\right)$. The linearized collision operator $\mathbf{L}$ is nonpositive, self-adjoint and its kernel is the subspace of five dimensions generated by

$$
\mathcal{N}=\operatorname{ker} \mathbf{L}=\operatorname{span}\left\{\sqrt{\mu} ; v_{i} \sqrt{\mu}, i=1,2,3 ;|v|^{2} \sqrt{\mu}\right\} .
$$

Let $\mathbf{P}_{0}$ be the projection operator from $L^{2}\left(\mathbb{R}_{v}^{3}\right)$ to the subspace $\mathcal{N}$ and $\mathbf{P}_{1}=\mathbf{I}-\mathbf{P}_{0}$. Then there exists a constant $\sigma>0$ such that

$$
-\int_{\mathbb{R}^{3}} f \mathbf{L} f d v \geq \sigma \int_{\mathbb{R}^{3}} \nu(v)\left(\mathbf{P}_{1} f\right)^{2} d v, \quad f \in D(\mathbf{L}) .
$$


Moreover, the bilinear collision operator $\Gamma(f, g)$ is given by

$$
\begin{aligned}
\Gamma(f, g)= & \mu^{-\frac{1}{2}} \mathcal{Q}\left(\mu^{\frac{1}{2}} f, \mu^{\frac{1}{2}} g\right)=\Gamma_{\text {gain }}(f, g)-\Gamma_{\text {loss }}(f, g) \\
= & \int_{\mathbb{R}^{3}} \int_{\mathbb{S}^{2}}|u-v|^{\gamma} \mu^{\frac{1}{2}}(u) f\left(u^{\prime}\right) g\left(v^{\prime}\right) B(\theta) d u d \omega \\
& -\left[\int_{\mathbb{R}^{3}} \int_{\mathbb{S}^{2}}|u-v|^{\gamma} \mu^{\frac{1}{2}}(u) f(u) B(\theta) d u d \omega\right] g(v) .
\end{aligned}
$$

Before starting the main result, we introduce a function space below. Let $\langle\cdot, \cdot\rangle$ denote the standard $L^{2}$ inner product in $\mathbb{R}^{3}$, with its $L^{2}$ norm given by $|\cdot|_{2}$. We define a weighed $L^{2}$ norm as

$$
|g|_{\nu}^{2}=\langle\nu(v) g, g\rangle .
$$

Moreover, $(\cdot, \cdot)$ is the $L^{2}$ inner product in $\mathbb{R}^{3} \times \mathbb{R}^{3}$ with its $L^{2}$ norm denoted by $\|\cdot\|$. A similar weighted norm is defined as

$$
\|g\|_{\nu}^{2}=(\nu(v) g, g) .
$$

We introduce a weighted function of $v$ as $w(v)=(1+|v|)^{\gamma}$. Letting $\alpha=\left[\alpha_{0}, \alpha_{1}, \alpha_{2}, \alpha_{3}\right]$ and $\beta=\left[\beta_{1}, \beta_{2}, \beta_{3}\right]$, we denote

$$
\partial^{\alpha}=\partial_{t}^{\alpha_{0}} \partial_{x_{1}}^{\alpha_{1}} \partial_{x_{2}}^{\alpha_{2}} \partial_{x_{3}}^{\alpha_{3}}, \partial_{\beta}=\partial_{v_{1}}^{\beta_{1}} \partial_{v_{2}}^{\beta_{2}} \partial_{v_{3}}^{\beta_{3}}, \partial_{\beta}^{\alpha}=\partial_{t}^{\alpha_{0}} \partial_{x_{1}}^{\alpha_{1}} \partial_{x_{2}}^{\alpha_{2}} \partial_{x_{3}}^{\alpha_{3}} \partial_{v_{1}}^{\beta_{1}} \partial_{v_{2}}^{\beta_{2}} \partial_{v_{3}}^{\beta_{3}} .
$$

Let $N$ be a positive integer, and let

$$
\begin{gathered}
\left.\||| f(t)\|\right|^{2}=\sum_{|\alpha|+|\beta| \leq N}\left\|w^{|\beta|} \partial_{\beta}^{\alpha} f(t)\right\|^{2}, \\
\mid\|f(t)\|\left\|_{\nu}^{2}=\sum_{|\beta| \leq N}\right\| w^{|\beta|} \partial_{\beta} \mathbf{P}_{1} f(t)\left\|_{\nu}^{2}+\sum_{|\alpha|+|\beta| \leq N, \alpha>0}\right\| w^{|\beta|} \partial_{\beta}^{\alpha} f(t) \|_{\nu}^{2}, \\
\left|\|f(t) \mid\|_{F P}^{2}=\sum_{|\alpha|+|\beta| \leq N, \alpha>0}\left(\left\|w^{|\beta|} \nabla_{v} \partial_{\beta}^{\alpha} f(t)\right\|^{2}+\left\||v| w^{|\beta|} \partial_{\beta}^{\alpha} f(t)\right\|^{2}\right) .\right.
\end{gathered}
$$

We define the high order energy norm as

$$
\mathcal{E}(f(t))=\left|\|f(t) \mid\|^{2}+\int_{0}^{t}\left(\left|\left\|f ( s ) \left|\left\|_{\nu}^{2}+\varepsilon\left|\|f(s) \mid\|_{F P}^{2}\right) d s,\right.\right.\right.\right.\right.\right.
$$

with the initial energy

$$
\mathcal{E}\left(f_{0}\right)=\mathcal{E}(f(0))=\|\| f_{0} \|\left.\right|^{2} .
$$

Now we introduce the space $Z_{1}=L_{v}^{2}\left(L_{x}^{1}\right)$ :

$$
Z_{1}=\left\{f:\|f\|_{Z_{1}}=\left(\int_{\mathbb{R}^{3}}\left(\int_{\mathbb{R}^{3}}|f(x, v)| d x\right)^{2} d v\right)^{\frac{1}{2}}<\infty\right\} .
$$

The space $Z_{1}$ is used to control the lower frequency of the solution $f$ to (1.5) after taking the Fourier transform with respect to the spatial variable, so as to establish its long time behavior. By taking the Fourier transform to the FPB equation with respect to the spatial variables and making use of the compensating function, we are able to control the low frequency part in terms of the $Z_{1}$ norm of initial data $f_{0}$, and we obtain the expected long time decay rates of global solutions (refer to Section 4 for details).

Throughout this paper, we assume that $N \geq 8$. We have the main results as follows. 
Theorem 1.1. Let $F_{0}(x, v)=\mu+\sqrt{\mu} f_{0}(x, v)$. Then, there exist small constants $\delta>$ $0, \varepsilon_{0}>0$ such that if $\mathcal{E}\left(f_{0}\right) \leq \delta$ and $\varepsilon \in\left(0, \varepsilon_{0}\right]$, the solution $f$ of the initial value problem (1.5) - (1.6) exists globally in time and satisfies

$$
\sup _{0 \leq t \leq \infty} \mathcal{E}(f(t)) \leq C \mathcal{E}\left(f_{0}\right)
$$

where $C>0$ is a constant. Moreover, if $f_{0} \in Z_{1}$, then for any $t>0$

$$
\||f(t)|\| \mid \leq C\left(\left|\left\|f_{0}\right\|\right| \mid+\left\|f_{0}\right\|_{Z_{1}}\right)(1+\sqrt{\varepsilon} t)^{-\frac{3}{4}} .
$$

REMARK 1.2. It is easy to verify that if $f_{0}=e^{-\frac{|x|^{2}}{2}} \sqrt{\mu}$, then $f_{0} \in Z_{1}$ and $\mathcal{E}\left(f_{0}\right)<\infty$. There has been much rigorous analysis established recently about the FPB equation (1.1). For instance, the zero diffusion limit $\varepsilon \rightarrow 0_{+}$was investigated by Hamdache when a solution is a perturbation of a vacuum state [18. The global existence theory of IVP (1.1)-(1.2) was proven by DiPerna and Lions [10 in the $L^{1}$ framework for the renormalized solution and was obtained by Hamdache in terms of a direct construction near the vacuum state [17. On the other hand, the global existence of classical solutions of IVP (1.1)-(1.2) for the hard sphere collision, where the Fokker-Planck operator is given by $L_{F P} f=\Delta f$, was established by Li and Matsumura [21, 22, for initial datum near an absolute Maxwellian, where it is shown that the global strong solution tends time-asymptotically to an self-similar Maxwellian with respect to time and velocity for a small perturbation of an absolute Maxwellian. Zhong and Li 32 obtained the global smooth solution starting from the absolute Maxwellian and its long time decay rate of the FPB equation for the collision of hard spheres or hard potentials.

There has recently been much important progress on the global existence and long time behavior for the Boltzmann equation (with hard potential $0<\gamma<1$ or hard sphere $\gamma=1$ ); refer to [9, 12, 13, 14, 23, 24, 25. For the case of soft potentials $-3<\gamma<0$, there have also been important results; for instance, Caflisch [5, 6] established exponential decay as well as global in time solutions for the Boltzmann equation near the Maxwellian with $-1<\gamma<0$ in a periodic box by use of spectral analysis. Guo [16 generalized the results in [6] to the cases $-3<\gamma<0$ by an energy method developed in [14, 15] and established the decay rate of $e^{-\lambda t^{p}}$ for some $\lambda>0$ and $0<p<1$ in [28]. In the whole space, it was Ukai and Asano [30] who obtained the decay rate $O\left(t^{-\alpha}\right)$ with $0<\alpha<1$ and the global solutions of the Boltzmann equation near the global Maxwellian with $-1<\gamma<0$, where their optimal case in $\mathbb{R}^{3}$ yields $\alpha=3 / 4$. Hsiao and $\mathrm{Yu}$ [19] obtained the global solutions of the Boltzmann and Landau equations near the Maxwellian with $-3 \leq \gamma<0$. By a completely different approach, Desvillettes and Villani [8] have recently developed a framework to study the convergence to Maxwellians for general smooth solutions, which as an application can lead to the almost exponential decay rates (i.e., faster than any given polynomial) for smooth solutions to the cutoff soft potential Boltzmann equation and the Landau equation. Yang and Yu 31] established the long time behavior of the relativistic Boltzmann and Landau equations. Recently, Strain established the optimal time decay rates of classical solutions to the hard and soft potential Boltzmann equation in the whole space without the angular cut-off assumption [29]. 
For the Fokker-Planck equation, the explicit estimates of relaxation to equilibrium could be obtained by the direct study of the dissipation of entropy with the help of logarithmic Sobolev inequalities. A detailed analysis of entropy production inequalities for linear Fokker-Planck type equations was performed [1, 3, 27. The algebraic decay towards the global equilibrium for the linear spatially inhomogeneous Fokker-Planck equation in a confining potential was obtained in 7 . The construction of the global classical solutions is based on the energy method developed by Guo 13, 14, 15, 16, for the Boltzmann equation and related kinetic models. The long time behavior is established by taking the Fourier transform to the FPB equation (1.5) in spatial variables, then making use of the compensating function [11,20] to gain an energy inequality, and finally applying Gronwall's inequality to obtain an algebraic decay.

The rest of the paper is arranged as follows. In Section 2, we reformulate the original FPB equation and decompose it into the macroscopic part and the microscopic part respectively. Then we establish the a priori estimates on them. In Section 3, the global existence of the strong solution to the IVP problem for the FPB equation is shown, and finally the large time behavior of the global solution is obtained in Section 4 .

\section{The a priori estimates}

We decompose the distribution function $f$ into the microscopic part and the macroscopic part as 13

$$
f=\mathbf{P}_{0} f+\mathbf{P}_{1} f,
$$

where the macroscopic part $\mathbf{P}_{0} f$ is defined as the linear combination of the basis in $k e r \mathbf{L}$

$$
\mathbf{P}_{0} f=\left\{a(t, x)+\sum_{j=1}^{3} b_{j}(t, x) v_{j}+c(t, x)|v|^{2}\right\} \sqrt{\mu} .
$$

The FPB equation (1.5) can be decomposed as

$$
\left\{\partial_{t}+v \cdot \nabla_{x}-\mathbf{F}\right\} \mathbf{P}_{0} f=-\left\{\partial_{t}+v \cdot \nabla_{x}+\mathbf{L}-\mathbf{F}\right\} \mathbf{P}_{1} f+\Gamma(f, f) .
$$

We can derive the macroscopic equations for coefficients $a(t, x), b_{j}(t, x), c(t, x)$ of $\mathbf{P}_{0} f$ as follows. In fact, from (1.7), we have

$$
\mathbf{F}(\sqrt{\mu})=0, \quad \mathbf{F}\left(v_{i} \sqrt{\mu}\right)=-v_{i} \sqrt{\mu}, \quad \mathbf{F}\left(|v|^{2} \sqrt{\mu}\right)=6 \sqrt{\mu}-2|v|^{2} \sqrt{\mu} .
$$

Then the left-hand side of (2.2) becomes

$$
\begin{gathered}
\left\{\left(\partial_{t} a-6 \varepsilon c\right)+\sum_{j=1}^{3} v_{j}\left(\partial_{x_{j}} a+\partial_{t} b_{j}+\varepsilon b_{j}\right)+\sum_{j>i}\left(\partial_{x_{j}} b_{i}+\partial_{x_{i}} b_{j}\right) v_{i} v_{j}\right. \\
\left.+\sum_{j=1}^{3}\left(\partial_{t} c+\partial_{x_{j}} b_{j}+2 \varepsilon c\right) v_{j}^{2}+\sum_{j=1}^{3} v_{j}|v|^{2} \partial_{x_{j}} c\right\} \sqrt{\mu} .
\end{gathered}
$$

For fixed $(t, x)$, there is an expansion of left-hand side terms of (2.2) with respect to the following 13 moments:

$$
v_{i}|v|^{2} \sqrt{\mu}, v_{i}^{2} \sqrt{\mu}, v_{i} v_{j} \sqrt{\mu}, v_{i} \sqrt{\mu}, \sqrt{\mu}, \quad 1 \leq i<j \leq 3 .
$$


With the help of them, we can obtain the macroscopic equations for $a(t, x), b(t, x), c(t, x)$ as

$$
\begin{aligned}
& \partial_{t} a-6 \varepsilon c=l_{a}+h_{a}, \\
& \partial_{x_{i}} a+\partial_{t} b_{i}+\varepsilon b_{i}=l_{b i}+h_{b i}, \\
& \partial_{x_{j}} b_{i}+\partial_{x_{j}} b_{j}=l_{i j}+h_{i j}, \quad i \neq j, \\
& \partial_{t} c+\partial_{x_{i}} b_{i}+2 \varepsilon c=l_{i}+h_{i}, \\
& \partial_{x_{i}} c=l_{c i}+h_{c i},
\end{aligned}
$$

where $l_{c i}(t, x), l_{i}(t, x), l_{i j}(t, x), l_{b i}(t, x)$ and $l_{a}(t, x)$ are the coefficients with respect to (2.4) for the linear term $-\left\{\partial_{t}+v \cdot \nabla_{x}+\mathbf{L}-\mathbf{F}\right\} \mathbf{P}_{1} f$, while $h_{c i}(t, x), h_{i}(t, x), h_{i j}(t, x), h_{b i}(t, x)$ and $h_{a}(t, x)$ are the corresponding coefficients for $\Gamma(f, f)$.

In the following, we will establish some a priori estimates so as to obtain the global existence and time decay rate of solutions for the FPB equation. First, we consider the nonlinear collision term $\Gamma(f, g)$. Notice that by a change of variable $u-v \rightarrow u$, it follows that

$$
\begin{aligned}
\partial_{\beta}^{\alpha} \Gamma(f, g)= & \partial_{\beta}^{\alpha}\left[\int_{\mathbb{R}^{3}} \int_{\mathbb{S}^{2}}|u|^{\gamma} e^{|u+v|^{2} / 2} f\left(v+u_{||}\right) g\left(v+u_{\perp}\right) B(\theta) d u d \omega\right] \\
& -\partial_{\beta}^{\alpha}\left[\int_{\mathbb{R}^{3}} \int_{\mathbb{S}^{2}}|u|^{\gamma} e^{|u+v|^{2} / 2} f(v+u) g(v) B(\theta) d u d \omega\right] \\
= & \sum C_{\beta}^{\beta_{0} \beta_{1} \beta_{2}} C_{\alpha}^{\alpha_{1} \alpha_{2}} \Gamma^{0}\left(\partial_{\beta_{1}}^{\alpha_{1}} f, \partial_{\beta_{2}}^{\alpha_{2}} g\right),
\end{aligned}
$$

where $\beta_{0}+\beta_{1}+\beta_{2}=\beta$ and $\alpha_{1}+\alpha_{2}=\alpha$. By the product rule and an inverse change of variables $u+v \rightarrow u$, it follows that

$$
\begin{aligned}
\Gamma^{0}\left(\partial_{\beta_{1}}^{\alpha_{1}} f, \partial_{\beta_{2}}^{\alpha_{2}} g\right)= & \int_{\mathbb{R}^{3}} \int_{\mathbb{S}^{2}}|u-v|^{\gamma} \partial_{\beta_{0}}\left(e^{-|u|^{2} / 2}\right) \partial_{\beta_{1}}^{\alpha_{1}} f\left(u^{\prime}\right) \partial_{\beta_{2}}^{\alpha_{2}} g\left(v^{\prime}\right) B(\theta) d u d \omega \\
& -\partial_{\beta_{2}}^{\alpha_{2}} g(v) \int_{\mathbb{R}^{3}} \int_{\mathbb{S}^{2}}|u-v|^{\gamma} \partial_{\beta_{0}}\left(e^{-|u|^{2} / 2}\right) \partial_{\beta_{1}}^{\alpha_{1}} f(u) B(\theta) d u d \omega \\
= & \Gamma_{\text {gain }}^{0}-\Gamma_{\text {loss }}^{0} .
\end{aligned}
$$

We have the following basic estimates in [14, 19]:

Lemma 2.1. There is a constant $C>0$ such that

$$
|\langle K f, g\rangle| \leq C|f|_{\nu}|g|_{\nu} .
$$

Let $|\beta|>0$ and $\theta \geq 0$. For any $\eta>0$, there exists a constant $C_{\eta}>0$ such that

$$
\left\langle\omega^{2 \theta} \partial_{\beta}(L f), \partial_{\beta} f\right\rangle \geq \frac{1}{2}\left|\omega^{\theta} \partial_{\beta} f\right|_{\nu}^{2}-\eta \sum_{\beta_{1} \leq \beta}\left|\omega^{\theta} \partial_{\beta_{1}} f\right|_{\nu}^{2}-C_{\eta}\left|\omega^{\theta} f\right|_{\nu}^{2} .
$$

Lemma 2.2. Recall (2.10) and let $\beta_{0}+\beta_{1}+\beta_{2}=\beta, \alpha_{1}+\alpha_{2}=\alpha,|\beta| \leq \theta$.

$$
\text { If }\left|\alpha_{1}\right|+\left|\beta_{1}\right| \leq N / 2 \text {, then }
$$

$$
\left|\left(\omega^{2 \theta} \Gamma^{0}\left(\partial_{\beta_{1}}^{\alpha_{1}} f, \partial_{\beta_{2}}^{\alpha_{2}} g\right), \partial_{\beta}^{\alpha} h\right)\right| \leq C\left[\sum_{\left|\alpha_{i}\right|+\left|\beta_{i}\right| \leq N}\left\|\omega^{\left|\beta_{i}\right|} \partial_{\beta_{i}}^{\alpha_{i}} f\right\|\right]\left\|\omega^{\theta} \partial_{\beta_{2}}^{\alpha_{2}} g\right\|_{\nu}\left\|\omega^{\theta} \partial_{\beta}^{\alpha} h\right\|_{\nu}
$$




$$
+C\left[\sum_{\left|\alpha_{i}\right|+\left|\beta_{1}\right| \leq N}\left\|\omega^{\left|\beta_{1}\right|} \partial_{\beta_{1}}^{\alpha_{i}} f\right\|\right]\left\|\omega^{\theta-\left|\beta_{2}\right|} \partial_{\beta_{2}}^{\alpha_{2}} g\right\|_{\nu}\left\|\omega^{\theta} \partial_{\beta}^{\alpha} h\right\|_{\nu} .
$$

If $\left|\alpha_{2}\right|+\left|\beta_{2}\right| \leq N / 2$, then

$$
\begin{array}{r}
\left|\left(\omega^{2 \theta} \Gamma^{0}\left(\partial_{\beta_{1}}^{\alpha_{1}} f, \partial_{\beta_{2}}^{\alpha_{2}} g\right), \partial_{\beta}^{\alpha} h\right)\right| \leq C\left[\sum_{\left|\alpha_{i}\right|+\left|\beta_{i}\right| \leq N}\left\|\omega^{\left|\beta_{i}\right|} \partial_{\beta_{i}}^{\alpha_{i}} g\right\|\right]\left\|\omega^{\theta} \partial_{\beta_{1}}^{\alpha_{1}} f\right\|_{\nu}\left\|\omega^{\theta} \partial_{\beta}^{\alpha} h\right\|_{\nu} \\
+C\left[\sum_{\left|\alpha_{i}\right|+\left|\beta_{2}\right| \leq N}\left\|\omega^{\left|\beta_{1}\right|} \partial_{\beta_{2}}^{\alpha_{i}} g\right\|\right]\left\|\omega^{\theta-\left|\beta_{2}\right|} \partial_{\beta_{1}}^{\alpha_{1}} f\right\|_{\nu}\left\|\omega^{\theta} \partial_{\beta}^{\alpha} h\right\|_{\nu} .
\end{array}
$$

Lemma 2.3. Let $f$ be the solution of the FPB equation (1.5). Then $\partial^{\alpha} \mathbf{P}_{0} f=\mathbf{P}_{0} \partial^{\alpha} f$, and

$$
\left\|\partial^{\alpha} \mathbf{P}_{0} f\right\|^{2}+\left\|\partial^{\alpha} \mathbf{P}_{1} f\right\|^{2}=\left\|\partial^{\alpha} f\right\|^{2} .
$$

There exists $C>1$ such that

$$
\frac{1}{C}\left\|\partial^{\alpha} \mathbf{P}_{0} f\right\|^{2} \leq\left\|\partial^{\alpha} a\right\|^{2}+\left\|\partial^{\alpha} \mathbf{b}\right\|^{2}+\left\|\partial^{\alpha} c\right\|^{2} \leq C\left\|\partial^{\alpha} \mathbf{P}_{0} f\right\|_{\nu}^{2}
$$

where $a, \mathbf{b}=\left(b_{1}, b_{2}, b_{3}\right), c$ are given by (2.1).

Lemma 2.4. Let $f$ be the solution of the FPB equation (1.5). Then

$$
\sum_{\alpha \leq N}\left\|\partial^{\alpha} h_{c i}\right\|+\left\|\partial^{\alpha} h_{i}\right\|+\left\|\partial^{\alpha} h_{i j}\right\|+\left\|\partial^{\alpha} h_{b i}\right\|+\left\|\partial^{\alpha} h_{a}\right\| \leq C|\|f\|\|\cdot\|| f \mid \|_{\nu}
$$

Lemma 2.5. Let $|\alpha|>0$ and $|\alpha|+|\beta| \leq N$. Then

$$
\left(\partial_{\beta}^{\alpha} \Gamma(f, f), \omega^{2|\beta|} \partial_{\beta}^{\alpha} f\right) \leq C|||f||| \cdot|||f| \|_{\nu}^{2} .
$$

Let $|\beta| \leq N$,. Then

$$
\begin{aligned}
(\Gamma(f, f), f) & \leq C\left|\left\|f \left|\left\|\cdot \left|\|f \mid\|_{\nu}^{2},\right.\right.\right.\right.\right. \\
\left(\partial_{\beta} \Gamma(f, f), \omega^{2|\beta|} \partial_{\beta} \mathbf{P}_{1} f\right) & \leq C\left|\left\|f \left|\left\|\cdot \left|\|f \mid\|_{\nu}^{2} .\right.\right.\right.\right.\right.
\end{aligned}
$$

Proof. For (2.18), from (2.13) and (2.14), it follows that

$$
\begin{aligned}
\left(\partial_{\beta}^{\alpha} \Gamma(f, f), \omega^{2|\beta|} \partial_{\beta}^{\alpha} f\right)= & \sum C_{\beta}^{\beta_{0} \beta_{1} \beta_{2}} C_{\alpha}^{\alpha_{1} \alpha_{2}}\left(\Gamma^{0}\left(\partial_{\beta_{1}}^{\alpha_{1}} f, \partial_{\beta_{2}}^{\alpha_{2}} f\right), \omega^{2|\beta|} \partial_{\beta}^{\alpha} f\right) \\
\leq & C \sum_{|\alpha|+|\beta| \leq N}\left\|\omega^{|\beta|} \partial_{\beta}^{\alpha} f\right\| \sum_{\left|\alpha_{2}\right|+\left|\beta_{2}\right|>N / 2}\left\|\omega^{\left|\beta_{2}\right|} \partial_{\beta_{2}}^{\alpha_{2}} f\right\|_{\nu}\left\|\omega^{|\beta|} \partial_{\beta}^{\alpha} f\right\|_{\nu} \\
& +C \sum_{|\alpha|+|\beta| \leq N}\left\|\omega^{|\beta|} \partial_{\beta}^{\alpha} f\right\| \sum_{\left|\alpha_{1}\right|+\left|\beta_{1}\right|>N / 2}\left\|\omega^{\left|\beta_{1}\right|} \partial_{\beta_{1}}^{\alpha_{1}} f\right\|_{\nu}\left\|\omega^{|\beta|} \partial_{\beta}^{\alpha} f\right\|_{\nu} \\
\leq & C\left|\left\|f \left|\|\cdot \cdot|| f \mid\|_{\nu}^{2} .\right.\right.\right.
\end{aligned}
$$

For (2.19) and (2.20), the details of the proof can be found in the proof of Theorem 1.1 in [19]. 
Lemma 2.6. Let $f$ be the solution to the FPB equation (1.5). Then for any $1 \leq i, j \leq 3$ it holds

$$
\sum_{\alpha \leq N-1}\left\|\partial^{\alpha} l_{c i}\right\|+\left\|\partial^{\alpha} l_{i}\right\|+\left\|\partial^{\alpha} l_{i j}\right\|+\left\|\partial^{\alpha} l_{b i}\right\|+\left\|\partial^{\alpha} l_{a}\right\| \leq C \sum_{\alpha \leq N}\left\|\mathbf{P}_{1} \partial^{\alpha} f\right\|_{\nu} .
$$

Proof. Let $e_{i}$ be one of the 13 moments defined in (2.4), and let $\lambda_{i j}=\left\langle e_{i}, e_{j}\right\rangle, 0 \leq$ $i, j \leq 13$. Assume that $\left\{a_{i j}\right\}_{13 \times 13}$ is the inverse matrix of $\left\{\lambda_{i j}\right\}_{13 \times 13}$. Notice that for fixed $(t, x)$, the coefficients $l_{c i}(t, x), l_{i}(t, x), l_{i j}(t, x), l_{b i}(t, x)$ and $l_{a}(t, x)$ take the form

$$
\sum_{j=1} a_{i j} \int-\left\{\partial_{t}+v \cdot \nabla_{x}+\mathbf{L}-\mathbf{F}\right\} \mathbf{P}_{1} f \cdot e_{i} d v .
$$

By Cauchy-Schwartz inequality, one has

$$
\left\|\left\langle\left\{\partial_{t}+v \cdot \nabla_{x}\right\} \partial^{\alpha} \mathbf{P}_{1} f, e_{i}\right\rangle\right\| \leq C\left(\left\|\mathbf{P}_{1} \partial_{t} \partial^{\alpha} f\right\|_{\nu}+\left\|\mathbf{P}_{1} \nabla_{x} \partial^{\alpha} f\right\|_{\nu}\right)
$$

and then by (2.11),

$$
\left\|\left\langle\mathbf{L}\left\{\mathbf{P}_{1}\right\} \partial^{\alpha} f, e_{i}\right\rangle\right\| \leq C\left\|\mathbf{P}_{1} \partial^{\alpha} f\right\|_{\nu} .
$$

From (1.7), it follows that

$$
\mathbf{F} f=\Delta_{v} f+\frac{3}{2} f-\frac{|v|^{2}}{4} f .
$$

Thus the operator $\mathbf{F}$ is self-adjoint. By (2.3), we have

$$
\left\|\left\langle\mathbf{F}\left(\mathbf{P}_{1} \partial^{\alpha} f\right), e_{i}\right\rangle\right\|=\left\|\left\langle\mathbf{P}_{1} \partial^{\alpha} f, \mathbf{F}\left(e_{i}\right)\right\rangle\right\| \leq C\left\|\mathbf{P}_{1} \partial^{\alpha} f\right\|_{\nu} .
$$

Combining (2.23)-(2.26), we obtain (2.22).

Lemma 2.7. Let $f(t, x, v)$ be a solution to the FPB equation (1.5). There exist positive constants $\delta, \sigma_{0}, C_{1}, C_{2}, C_{3}$ such that if \|\|$f(t)\|\|^{2} \leq \delta$, then

$$
\begin{array}{r}
\sum_{0<\alpha \leq N}\left(\mathbf{L} \partial^{\alpha} f, \partial^{\alpha} f\right)+C_{1} \frac{d}{d t} \int_{\mathbb{R}^{3}} \nabla \cdot \mathbf{b} a d x+C_{2} \varepsilon \int_{\mathbb{R}^{3}}\left(\mathbf{b}^{2}+c^{2}\right) d x \\
+C_{3}\left\|\mathbf{P}_{1} f\right\|_{\nu}^{2} \geq \sigma_{0} \sum_{0<\alpha \leq N}\left\|\partial^{\alpha} f\right\|_{\nu}^{2} .
\end{array}
$$

Proof. Since $(\mathbf{L} f, f) \geq \sigma\|\{\mathbf{I}-\mathbf{P}\} f\|_{\nu}^{2}$, it is sufficient to show that

$$
\sum_{0<\alpha \leq N}\left\|\mathbf{P}_{0} \partial^{\alpha} f\right\|_{\nu}^{2} \leq C \sum_{\alpha \leq N}\left\|\mathbf{P}_{1} \partial^{\alpha} f\right\|_{\nu}^{2}+C_{1} \frac{d}{d t} \int_{\mathbb{R}^{3}} \nabla \cdot \mathbf{b} a d x+C_{2} \varepsilon \int_{\mathbb{R}^{3}} \mathbf{b}^{2}+c^{2} d x .
$$

By (2.16), we only need to prove

$$
\begin{aligned}
& \sum_{0<\alpha \leq N}\left(\left\|\partial^{\alpha} a\right\|+\left\|\partial^{\alpha} \mathbf{b}\right\|+\left\|\partial^{\alpha} c\right\|\right)^{2} \leq C \sum_{\alpha \leq N}\left\|\mathbf{P}_{1} \partial^{\alpha} f\right\|_{\nu}^{2} \\
& \quad+C \sqrt{\delta}\left\{\sum_{0<\alpha \leq N}\left(\left\|\partial^{\alpha} a\right\|+\left\|\partial^{\alpha} \mathbf{b}\right\|+\left\|\partial^{\alpha} c\right\|\right)+\sum_{\alpha \leq N}\left\|\mathbf{P}_{1} \partial^{\alpha} f\right\|_{\nu}\right\}^{2} \\
& \quad+C_{1} \frac{d}{d t} \int_{\mathbb{R}^{3}} \nabla \cdot \mathbf{b} a d x+C_{2} \varepsilon \int_{\mathbb{R}^{3}} \mathbf{b}^{2}+c^{2} d x
\end{aligned}
$$


To prove (2.28), we first estimate $\nabla_{x} \partial^{\alpha} b$ with $|\alpha| \leq N-1$. Making use of the macroscopic equations (2.7) and (2.8), we have

$$
\begin{aligned}
\Delta_{x} \partial^{\alpha} b_{i}= & \left\{\sum_{j \neq i} \partial_{x_{j} x_{j}} \partial^{\alpha} b_{i}\right\}+\partial_{x_{i} x_{i}} \partial^{\alpha} b_{i} \\
= & \sum_{j \neq i}\left\{-\partial_{x_{i}} \partial^{\alpha} l_{j}-\partial_{x_{i}} \partial^{\alpha} h_{j}+\partial_{x_{i}} \partial^{\alpha} l_{i j}+\partial_{x_{j}} \partial^{\alpha} h_{i j}\right\} \\
& +\partial_{x_{i}} \partial^{\alpha} \partial_{t} c+2 \varepsilon \partial_{x_{i}} \partial^{\alpha} c-\partial_{x_{i}} \partial^{\alpha} l_{i}-\partial_{x_{i}} \partial^{\alpha} h_{i} \\
= & \sum_{j \neq i}\left\{-\partial_{x_{i}} \partial^{\alpha} l_{j}-\partial_{x_{i}} \partial^{\alpha} h_{j}+\partial_{x_{j}} \partial^{\alpha} l_{i j}+\partial_{x_{j}} \partial^{\alpha} h_{i j}\right\} \\
& -\partial_{x_{i} x_{i}} \partial^{\alpha} b_{i}+\partial_{x_{i}} \partial^{\alpha} l_{i}+\partial_{x_{i}} \partial^{\alpha} h_{i} .
\end{aligned}
$$

Therefore, taking the inner product between $\Delta_{x} \partial^{\alpha} b_{i}$ and $\partial^{\alpha} b_{i}$ over $L^{2}\left(\mathbb{R}^{3}\right)$ yields

$$
\left\|\nabla_{x} \partial^{\alpha} b_{i}\right\| \leq C \sum_{j \neq i}\left(\left\|\partial^{\alpha} l_{j}\right\|+\left\|\partial^{\alpha} h_{j}\right\|+\left\|\partial^{\alpha} l_{i j}\right\|+\left\|\partial^{\alpha} h_{i j}\right\|\right)+\left\|\partial^{\alpha} l_{i}\right\|+\left\|\partial^{\alpha} h_{i}\right\| .
$$

It follows from (2.8) and (2.9) that

$$
\begin{aligned}
\left\|\partial_{t} \partial^{\alpha} c\right\| & \leq\left\|\partial_{x_{i}} \partial^{\alpha} b_{i}\right\|+\left\|\partial^{\alpha} l_{i}\right\|+\left\|\partial^{\alpha} h_{i}\right\|+2 \varepsilon\left\|\partial^{\alpha} c\right\|, \\
\left\|\nabla_{x} \partial^{\alpha} c\right\| & \leq\left\|\partial^{\alpha} l_{c i}\right\|+\left\|\partial^{\alpha} h_{c i}\right\| .
\end{aligned}
$$

By (2.5) and (2.6),

$$
\begin{gathered}
\left\|\partial_{t} \partial^{\alpha} a\right\| \leq\left\|\partial^{\alpha} l_{a}\right\|+\left\|\partial^{\alpha} h_{a}\right\| \\
-\Delta_{x} \partial^{\alpha} a=\nabla_{x} \cdot\left(\partial_{t} \partial^{\alpha} \mathbf{b}+\varepsilon \partial^{\alpha} \mathbf{b}\right)-\sum_{i} \partial_{x_{i}} \partial^{\alpha}\left\{l_{b i}+h_{b i}\right\} .
\end{gathered}
$$

Taking the inner product between (2.33) and $\partial^{\alpha} a$, we have

$$
\left\|\nabla_{x} \partial^{\alpha} a\right\| \leq\left\|\partial_{t} \partial^{\alpha} \mathbf{b}\right\|+\varepsilon\left\|\partial^{\alpha} \mathbf{b}\right\|+\sum\left\|\partial^{\alpha}\left\{l_{b i}+h_{b i}\right\}\right\| .
$$

For $\alpha=0$, the same procedure yields

$$
\begin{aligned}
\left\|\nabla_{x} a\right\| & \leq \int_{\mathbb{R}^{3}} \nabla_{x} \cdot \partial_{t} \mathbf{b} a d x+\varepsilon\|\mathbf{b}\|+\left\|\left\{l_{b i}+h_{b i}\right\}\right\| \\
& =\frac{d}{d t} \int_{\mathbb{R}^{3}} \nabla_{x} \cdot \mathbf{b} a d x-\int_{\mathbb{R}^{3}} \nabla_{x} \cdot \mathbf{b} \partial_{t} a d x+\varepsilon\|\mathbf{b}\|+\left\|\left\{l_{b i}+h_{b i}\right\}\right\| \\
& \leq \frac{d}{d t} \int_{\mathbb{R}^{3}} \nabla_{x} \cdot \mathbf{b} a d x+\left\{\left\|\nabla_{x} \mathbf{b}\right\|+\left\|\partial_{t} a\right\|+\varepsilon\|\mathbf{b}\|+\left\|\left\{l_{b i}+h_{b i}\right\}\right\|\right\} .
\end{aligned}
$$

We finally estimate the time derivatives of $b_{i}(t, x)$. Taking the derivative $\partial_{t}^{j-1}$ in (2.6) with $0<j \leq N$, it follows that

$$
\begin{aligned}
\left\|\partial_{t}^{j} b_{i}\right\|^{2} & =\left\|-\partial_{x_{i}} \partial_{t}^{j-1} a+\varepsilon \partial_{t}^{j-1} b_{i}+\partial_{t}^{j-1}\left\{l_{b i}+h_{b i}\right\}\right\|^{2} \\
& \leq 3\left\|\partial_{x_{i}} \partial_{t}^{j-1} a\right\|^{2}+3 \varepsilon\left\|\partial_{t}^{j-1} b_{i}\right\|+3\left\|\partial_{t}^{j-1}\left\{l_{b i}+h_{b i}\right\}\right\|^{2} .
\end{aligned}
$$

By (2.29) - 2.36), we obtain (2.28).

Making use of ideas similar to those proving Theorem 3 in [14, we have 
Lemma 2.8. Let $\left|\alpha_{1}\right|+\left|\alpha_{2}\right|=N$.

$$
\begin{aligned}
& \left\|\nu^{-\frac{1}{2}} \Gamma^{0}\left(\partial^{\alpha_{1}} f, \partial^{\alpha_{2}} g\right)\right\|^{2} \leq C\left[\sum_{|\alpha|+|\beta| \leq N}\left\|\omega^{|\beta|} \partial_{\beta}^{\alpha} f\right\|^{2}\right]\left\|\partial^{\alpha_{2}} g\right\|_{\nu}^{2}, \text { if }\left|\alpha_{1}\right| \leq N / 2 ; \\
& \left\|\nu^{-\frac{1}{2}} \Gamma^{0}\left(\partial^{\alpha_{1}} f, \partial^{\alpha_{2}} g\right)\right\|^{2} \leq C\left[\sum_{|\alpha|+|\beta| \leq N}\left\|\omega^{|\beta|} \partial_{\beta}^{\alpha} g\right\|^{2}\right]\left\|\partial^{\alpha_{1}} f\right\|_{\nu}^{2}, \text { if }\left|\alpha_{1}\right| \geq N / 2 .
\end{aligned}
$$

Moreover,

$$
\left\|\nu^{-\frac{1}{2}} \Gamma(f, g)\right\|_{Z_{1}}^{2} \leq C\left[\sum_{|\beta| \leq 2}\left\|\omega^{|\beta|} \partial_{\beta} f\right\|^{2}\right]\|g\|_{\nu}^{2} .
$$

Proof. From (2.13) and (2.14) with $\theta=0$, we have

$$
\begin{aligned}
& \left\|\nu^{-\frac{1}{2}} \Gamma^{0}\left(\partial^{\alpha_{1}} f, \partial^{\alpha_{2}} g\right)\right\|^{2}=\left(\Gamma^{0}\left(\partial^{\alpha_{1}} f, \partial^{\alpha_{2}} g\right), \nu^{-1} \Gamma^{0}\left(\partial^{\alpha_{1}} f, \partial^{\alpha_{2}} g\right)\right) \\
& \leq C\left[\sum_{|\alpha|+|\beta| \leq N}\left\|\omega^{|\beta|} \partial_{\beta}^{\alpha} f\right\|\right]\left\|\partial^{\alpha_{2}} g\right\|_{\nu}\left\|\nu^{-1} \Gamma^{0}\left(\partial^{\alpha_{1}} f, \partial^{\alpha_{2}} g\right)\right\|_{\nu} \\
& \quad=C\left[\sum_{|\alpha|+|\beta| \leq N}\left\|\omega^{|\beta|} \partial_{\beta}^{\alpha} f\right\|\right]\left\|\partial^{\alpha_{2}} g\right\|_{\nu}\left\|\nu^{-\frac{1}{2}} \Gamma^{0}\left(\partial^{\alpha_{1}} f, \partial^{\alpha_{2}} g\right)\right\|
\end{aligned}
$$

where we assume that $\left|\alpha_{1}\right| \leq N / 2$. Therefore, we obtain (2.37).

Now we deal with (2.38). Since $\Gamma(f, g)=\Gamma_{\text {gain }}(f, g)-\Gamma_{\text {loss }}(f, g)$, we have

$$
\left\|\nu^{-\frac{1}{2}} \Gamma(f, g)\right\|_{Z_{1}} \leq\left\|\nu^{-\frac{1}{2}} \Gamma_{\text {loss }}(f, g)\right\|_{Z_{1}}+\left\|\nu^{-\frac{1}{2}} \Gamma_{\text {gain }}(f, g)\right\|_{Z_{1}} .
$$

First we consider the loss term $\Gamma_{\text {loss }}(f, g)$, which is bounded by

$$
\begin{aligned}
& \int \nu^{-1}\left(\int B(\theta)|u-v|^{\gamma} e^{-\frac{|u|^{2}}{4}}|f(u) g(v)| d u d x\right)^{2} d v \\
& \leq C \int \nu^{-1}\left(\int|u-v|^{2 \gamma} e^{-\frac{|u|^{2}}{2}} d u\right)\left(\int f^{2}(u) d x d u\right)\left(\int g^{2}(v) d x\right) d v \\
& \leq C \int f^{2}(u) d x d u \int \nu g^{2}(v) d x d v .
\end{aligned}
$$

Then we consider the gain term $\Gamma_{\text {gain }}(f, g)$. Split the integration domain into two parts:

$$
\{|u| \geq|v| / 2\} \cup\{|u| \leq|v| / 2\} .
$$

For the first part, $\{|u| \geq|v| / 2\}$, it is obvious that

$$
e^{-\frac{|u|^{2}}{4}} \leq e^{-\frac{|u|^{2}}{8}} e^{-\frac{|v|^{2}}{32}} .
$$

Hence, the integral of $\Gamma_{\text {gain }}(f, g)$ over $\{|u| \geq|v| / 2\}$ is bounded by

$$
\begin{aligned}
& \int_{|u| \geq|v| / 2} \nu^{-1}\left(\int B(\theta)|u-v|^{\gamma} e^{-\frac{|u|^{2}}{4}}\left|f\left(u^{\prime}\right) g\left(v^{\prime}\right)\right| d u d \omega d x\right)^{2} d v \\
& \leq C \int \nu^{-1} \int|u-v|^{\gamma} e^{-\frac{|u|^{2}}{4}} d u \int|u-v|^{\gamma} e^{-\frac{|u|^{2}}{4}}\left\|f\left(u^{\prime}\right)\right\|_{L_{x}^{2}}^{2}\left\|g^{2}\left(v^{\prime}\right)\right\|_{L_{x}^{2}}^{2} d u d v
\end{aligned}
$$




$$
\leq C \int\left|u^{\prime}-v^{\prime}\right|^{\gamma} e^{-\frac{\left|u^{\prime}\right|^{2}}{8}-\frac{\left|v^{\prime}\right|^{2}}{32}}\left\|f\left(u^{\prime}\right)\right\|_{L_{x}^{2}}^{2}\left\|g^{2}\left(v^{\prime}\right)\right\|_{L_{x}^{2}}^{2} d u^{\prime} d v^{\prime} .
$$

Since

we have

$$
\sup _{u^{\prime}}\left\{e^{-\frac{\left|u^{\prime}\right|^{2}}{32}}\left\|f\left(u^{\prime}\right)\right\|_{L_{x}^{2}}^{2}\right\} \leq C \sum_{|\beta| \leq 2}\left\|\omega^{|\beta|} \partial_{\beta} f\right\|^{2},
$$

$$
\int_{|u| \geq|v| / 2} \nu^{-1}\left(\int\left|\Gamma_{\text {gain }}(f, g)\right| d x\right)^{2} d v \leq C \sum_{|\beta| \leq 2}\left\|\omega^{|\beta|} \partial_{\beta} f\right\|^{2} \times\|g\|_{\nu}^{2} .
$$

Now we consider the second part, $\{|u| \leq|v| / 2\}$. It is obvious that

$$
|v-u| \geq|v|-|u| \geq|v| / 2 .
$$

Since $\gamma<0$, this implies that $|u-v|^{\gamma} \leq C|v|^{\gamma}$. Then, the integral of $\Gamma_{\text {gain }}(f, g)$ over $\{|u| \leq|v| / 2\}$ is bounded by

$$
\begin{aligned}
& \int_{|u| \leq|v| / 2} \nu^{-1}\left(\int B(\theta)|u-v|^{\gamma} e^{-\frac{|u|^{2}}{4}}\left|f\left(u^{\prime}\right) g\left(v^{\prime}\right)\right| d u d \omega d x\right)^{2} d v \\
& \leq C \int \nu^{-1} \int|u-v|^{\gamma} e^{-\frac{|u|^{2}}{4}} d u \int|u-v|^{\gamma} e^{-\frac{|u|^{2}}{4}}\left\|f\left(u^{\prime}\right)\right\|_{L_{x}^{2}}^{2}\left\|g^{2}\left(v^{\prime}\right)\right\|_{L_{x}^{2}}^{2} d u d v \\
& \leq C \int|v|^{\gamma}\left\|f\left(u^{\prime}\right)\right\|_{L_{x}^{2}}^{2}\left\|g^{2}\left(v^{\prime}\right)\right\|_{L_{x}^{2}}^{2} d u^{\prime} d v^{\prime} .
\end{aligned}
$$

Note that $|u| \leq|v| / 2$. From (1.4), we have

$$
\left|u^{\prime}\right|+\left|v^{\prime}\right| \leq C(|u|+|v|) \leq C|v| .
$$

For $\gamma<0$, the above implies

$$
|v|^{\gamma} \leq C\left|u^{\prime}\right|^{\gamma}, \quad|v|^{\gamma} \leq C\left|v^{\prime}\right|^{\gamma} .
$$

We split the domain $\{|u| \leq|v| / 2\}$ into $\{|u| \leq|v| / 2$ and $|v| \geq 1\}$ and $\{|u| \leq|v| / 2$ and $|v| \leq$ $1\}$. For the first part, by (2.45), we have

$$
\int_{|u| \leq|v| / 2,|v| \geq 1} \leq C \int\left(1+\left|v^{\prime}\right|\right)^{\gamma}\left\|f\left(u^{\prime}\right)\right\|_{L_{x}^{2}}^{2}\left\|g\left(v^{\prime}\right)\right\|_{L_{x}^{2}}^{2} d u^{\prime} d v^{\prime} \leq C\|f\|^{2}\|g\|_{\nu}^{2} .
$$

For the second part, by (2.44) and (2.45), it follows that

$$
\begin{aligned}
\int_{|u| \leq|v| / 2,|v| \leq 1} & \leq \int_{\left|u^{\prime}\right| \leq C,\left|v^{\prime}\right| \leq C}\left|u^{\prime}\right|^{\gamma}\left\|f\left(u^{\prime}\right)\right\|_{L_{x}^{2}}^{2}\left\|g\left(v^{\prime}\right)\right\|_{L_{x}^{2}}^{2} d u^{\prime} d v^{\prime} \\
& \leq C \sup _{\left|u^{\prime}\right| \leq C}\|f\|_{L_{x}^{2}}^{2} \int_{\left|u^{\prime}\right| \leq C}\left|u^{\prime}\right|^{\gamma} d u^{\prime} \int_{\left|v^{\prime}\right| \leq C} g^{2}\left(v^{\prime}\right) d v^{\prime} d x .
\end{aligned}
$$

Since $\gamma \geq-1$, the above implies that

$$
\int_{|u| \leq|v| / 2,|v| \leq 1} \leq C \sum_{|\beta| \leq 2}\left\|\omega^{|\beta|} \partial_{\beta} f\right\|^{2} \times\|g\|_{\nu}^{2} .
$$

Thus we obtain (2.38). This completes the proof.

Now, let us turn to the estimates of the Fokker-Planck operator F. We have 
LEmma 2.9. Let $f$ be a solution to the FPB equation (1.5), i.e.,

$$
-\langle\mathbf{F} f, f\rangle=\left|\nabla_{v} f+\frac{v}{2} f\right|_{2}^{2} \geq(2 \pi)^{3 / 2}\left[\mathbf{b}(t, x)^{2}+c(t, x)^{2}\right] .
$$

Moreover, if $\varepsilon>0$ is small enough, then

$$
\begin{aligned}
\left|\partial^{\alpha} f\right|_{\nu}^{2}-\varepsilon\left\langle\mathbf{F} \partial^{\alpha} f, \partial^{\alpha} f\right\rangle \geq & \frac{\varepsilon}{2}\left|\nabla_{v} \partial^{\alpha} f\right|_{2}^{2}+\frac{\varepsilon}{8}\left|v \partial^{\alpha} f\right|_{2}^{2}+C \sqrt{\varepsilon}\left|\partial^{\alpha} f\right|_{2}^{2} \\
\left|\omega \partial_{\beta}^{\alpha} f\right|_{\nu}^{2}-\varepsilon\left\langle\partial_{\beta} \mathbf{F} \partial^{\alpha} f, \omega^{2} \partial_{\beta}^{\alpha} f\right\rangle \geq & \frac{\varepsilon}{2}\left|\omega \nabla_{v} \partial_{\beta}^{\alpha} f\right|_{2}^{2}+\frac{\varepsilon}{8}\left|v \omega \partial_{\beta}^{\alpha} f\right|_{2}^{2}+C \sqrt{\varepsilon}\left|\omega \partial_{\beta}^{\alpha} f\right|_{2}^{2} \\
& -C \varepsilon\left|\partial^{\alpha} f\right|_{2}^{2}, \quad \text { if }|\beta|=1 \\
\left|\omega^{|\beta|} \partial_{\beta}^{\alpha} f\right|_{\nu}^{2}-\varepsilon\left\langle\partial_{\beta} \mathbf{F} \partial^{\alpha} f, \omega^{2|\beta|} \partial_{\beta}^{\alpha} f\right\rangle \geq & \frac{\varepsilon}{2}\left|\omega^{|\beta|} \nabla_{v} \partial_{\beta}^{\alpha} f\right|_{2}^{2}+\frac{\varepsilon}{8}\left|v \omega^{|\beta|} \partial_{\beta}^{\alpha} f\right|_{2}^{2}+C \sqrt{\varepsilon}\left|\omega^{|\beta|} \partial_{\beta}^{\alpha} f\right|_{2}^{2} \\
& -C \varepsilon\left(\left|\omega^{|\beta|-1} \partial_{\beta-1}^{\alpha} f\right|_{2}^{2}+\left|\omega^{|\beta|-2} \partial_{\beta-2}^{\alpha} f\right|_{2}^{2}\right), \text { if }|\beta| \geq 2 .
\end{aligned}
$$

Proof. Denote

$$
\psi_{0}=\sqrt{\mu}, \psi_{i}=v_{i} \sqrt{\mu}, i=1,2,3, \psi_{4}=|v|^{2} \sqrt{\mu} .
$$

By (2.3), we have

and

$$
\int_{\mathbb{R}^{3}} \mathbf{F P}_{1} f \psi_{i} d v=\int_{\mathbb{R}^{3}} \mathbf{P}_{1} f \mathbf{F} \psi_{i} d v=0
$$

$$
\begin{gathered}
\mathbf{P}_{0} \mathbf{F} f=\mathbf{P}_{0} \mathbf{F}\left(\mathbf{P}_{0} f\right)+\mathbf{P F}\left(\mathbf{P}_{1} f\right)=\mathbf{F} \mathbf{P}_{0} f, \\
\mathbf{P}_{1}(\mathbf{F} f)=\mathbf{F} f-\mathbf{P}_{0}(\mathbf{F} f)=\mathbf{F}\left(\mathbf{P}_{1} f\right) .
\end{gathered}
$$

With the help of above properties, we can obtain

$$
\begin{aligned}
\langle\mathbf{F} f, f\rangle & =\left\langle\mathbf{F}\left(\mathbf{P}_{0} f\right)+\mathbf{F}\left(\mathbf{P}_{1} f\right), \mathbf{P}_{0} f+\mathbf{P}_{1} f\right\rangle \\
& =\left\langle\mathbf{F}\left(\mathbf{P}_{0} f\right), \mathbf{P}_{0} f\right\rangle+\left\langle\mathbf{F}\left(\mathbf{P}_{1} f\right), \mathbf{P}_{1} f\right\rangle,
\end{aligned}
$$

which implies

$$
-\langle\mathbf{F} f, f\rangle \geq\left|\left(\nabla_{v}+v / 2\right) \mathbf{P}_{0} f\right|_{2}^{2}=|\mathbf{b}(t, x) \sqrt{\mu}+2 c(t, x) v \sqrt{\mu}|_{2}^{2}=(2 \pi)^{3 / 2}\left(\mathbf{b}^{2}+12 c^{2}\right) .
$$

The estimate (2.50) (2.52) can be shown by a similar argument. We only deal with (2.52) for simplicity. By (2.25), we have

$$
-\left\langle\partial_{\beta} \mathbf{F} \partial^{\alpha} f, \omega^{2|\beta|} \partial_{\beta}^{\alpha} f\right\rangle=-\left\langle\Delta_{v} \partial_{\beta}^{\alpha} f, \omega^{2|\beta|} \partial_{\beta}^{\alpha} f\right\rangle+\frac{3}{2}\left|\omega^{|\beta|} \partial_{\beta}^{\alpha} f\right|_{2}^{2}-\left\langle\partial_{\beta}\left(\frac{|v|^{2}}{4} \partial^{\alpha} f\right), \omega^{2|\beta|} \partial_{\beta}^{\alpha} f\right\rangle .
$$

The right-hand side terms of (2.55) can be estimated respectively as follows. Due to the fact that

$$
\left|\partial_{v}(1+|v|)^{\gamma}\right|=\left|\gamma(1+|v|)^{\gamma-1} v_{i} /\right| v|| \leq C(1+|v|)^{\gamma}, \quad \text { for } \quad \gamma<0,
$$

we have by Cauchy-Schwartz inequality that

$$
\begin{aligned}
-\left\langle\Delta_{v} \partial_{\beta}^{\alpha} f, \omega^{2|\beta|} \partial_{\beta}^{\alpha} f\right\rangle & =\left\langle\nabla_{v} \partial_{\beta}^{\alpha} f, \omega^{2|\beta|} \nabla_{v} \partial_{\beta}^{\alpha} f\right\rangle+\left\langle\nabla_{v} \partial_{\beta}^{\alpha} f, \nabla_{v}\left(\omega^{2|\beta|}\right) \partial_{\beta}^{\alpha} f\right\rangle \\
& \geq \frac{1}{2}\left|\omega^{|\beta|} \nabla_{v} \partial_{\beta}^{\alpha} f\right|_{2}^{2}-C\left|\omega^{|\beta|} \partial_{\beta}^{\alpha} f\right|_{2}^{2} .
\end{aligned}
$$


Also, the third term is estimated as

$$
\begin{aligned}
& \frac{1}{4}\left\langle\partial_{\beta}\left(|v|^{2} \partial^{\alpha} f\right), \omega^{2|\beta|} \partial_{\beta}^{\alpha} f\right\rangle \\
& =\frac{1}{4}\left|v \omega^{|\beta|} \partial_{\beta}^{\alpha} f\right|_{2}^{2}+\frac{1}{4}\left\langle C_{\beta}^{\beta_{1}} \partial_{\beta_{1}}\left(|v|^{2}\right) \partial_{\beta-\beta_{1}}^{\alpha} f, \omega^{2|\beta|} \partial_{\beta}^{\alpha} f\right\rangle+\frac{1}{4}\left\langle C_{\beta}^{\beta_{2}} \partial_{\beta_{2}}\left(|v|^{2}\right) \partial_{\beta-\beta_{2}}^{\alpha} f, \omega^{2|\beta|} \partial_{\beta}^{\alpha} f\right\rangle \\
& \geq \frac{1}{8}\left|v \omega^{|\beta|} \partial_{\beta}^{\alpha} f\right|_{2}^{2}-C\left(\left|\omega^{|\beta|} \partial_{\beta}^{\alpha} f\right|_{2}^{2}+\left|\omega^{|\beta|-1} \partial_{\beta-1}^{\alpha} f\right|_{2}^{2}+\left|\omega^{|\beta|-2} \partial_{\beta-2}^{\alpha} f\right|_{2}^{2}\right),
\end{aligned}
$$

where $\left|\beta_{1}\right|=1,\left|\beta_{2}\right|=2$. Substituting (2.56)-2.57) into (2.55), we obtain

$$
\begin{aligned}
-\left\langle\partial_{\beta} \mathbf{F} \partial^{\alpha} f, \omega^{2|\beta|} \partial_{\beta}^{\alpha} f\right\rangle \geq & \frac{1}{2}\left|\omega^{|\beta|} \nabla_{v} \partial_{\beta}^{\alpha} f\right|_{2}^{2}+\frac{1}{8}\left|v \omega^{|\beta|} \partial_{\beta}^{\alpha} f\right|_{2}^{2} \\
& -C\left(\left|\omega^{|\beta|} \partial_{\beta}^{\alpha} f\right|_{2}^{2}+\left|\omega^{|\beta|-1} \partial_{\beta-1}^{\alpha} f\right|_{2}^{2}+\left|\omega^{|\beta|-2} \partial_{\beta-2}^{\alpha} f\right|_{2}^{2}\right) .
\end{aligned}
$$

Since

$$
\nu_{0}(1+|v|)^{\gamma} \leq|\nu(v)| \leq \nu_{1}(1+|v|)^{\gamma}, \quad-1 \leq \gamma<0,
$$

it follows that

$$
\left\langle\nu(v) \partial_{\beta}^{\alpha} f, \omega^{2|\beta|} \partial_{\beta}^{\alpha} f\right\rangle+\varepsilon\left|v \omega^{|\beta|} \partial_{\beta}^{\alpha} f\right|_{2}^{2} \geq C \sqrt{\varepsilon}\left|\omega^{|\beta|} \partial_{\beta}^{\alpha} f\right|_{2}^{2} .
$$

Combining (2.58) and (2.59), if $\varepsilon>0$ is small enough, we obtain (2.52).

\section{Global existence}

The following short time existence of classical solutions of IVP (1.5)-(1.6) can be established by performing the standard arguments as in [16, 21]. We omit the details. We have the following lemma.

Lemma 3.1. There exist $\delta>0$ and $T=T(\delta, \varepsilon)>0$ such that if $\mathcal{E}\left(f_{0}\right) \leq \delta$, then there is a unique classical solution $f=f(t, x, v)$ to (1.5)-(1.6) in $[0, T]$ satisfying

$$
\sup _{0 \leq t \leq T} \mathcal{E}(f(t)) \leq C \mathcal{E}\left(f_{0}\right)
$$

Based on the short time existence of the classical solution $f$ to IVP (1.5)-11.6), we can obtain global existence with the help of Lemmas 2.1 2.9 as follows.

Proof of global existence in Theorem 1.1. Assume that

$$
\delta_{1}=\sup _{0 \leq s \leq t} \mathcal{E}(f(s))
$$

is small enough. We take the inner product between $\partial^{\alpha}(\underline{1.5})$ and $\partial^{\alpha} f$ over $L^{2}\left(\mathbb{R}_{x}^{3} \times \mathbb{R}_{v}^{3}\right)$ to get

$$
\frac{1}{2} \frac{d}{d t} \sum_{\alpha>0}\left\|\partial^{\alpha} f\right\|^{2}+\sum_{\alpha>0}\left(L \partial^{\alpha} f, \partial^{\alpha} f\right)-\sum_{\alpha>0} \varepsilon\left(\mathbf{F} \partial^{\alpha} f, \partial^{\alpha} f\right)=\sum_{\alpha>0}\left(\partial^{\alpha} \Gamma(f, f), \partial^{\alpha} f\right) .
$$

We first deal with the case $0<\alpha \leq N$. By (2.18) and (2.27), we have

$$
\begin{aligned}
\frac{1}{2} & \frac{d}{d t}\left\{\sum_{\alpha>0}\left\|\partial^{\alpha} f\right\|^{2}-2 C_{1} \int_{\mathbb{R}^{3}} \nabla \cdot \mathbf{b} a d x\right\}+\sigma_{0} \sum_{\alpha>0}\left\|\partial^{\alpha} f\right\|_{\nu}^{2}-C_{2} \varepsilon \int_{\mathbb{R}^{3}} \mathbf{b}^{2}+c^{2} d x \\
- & C_{3}\left\|\mathbf{P}_{1} f\right\|_{\nu}^{2}+\varepsilon \sum_{\alpha>0}\left\|\nabla_{v} \partial^{\alpha} f+\frac{v}{2} \partial^{\alpha} f\right\|^{2} \leq C\|f\|\|\cdot\| f \|_{\nu}^{2}
\end{aligned}
$$


For the case $\alpha=0$, it follows from (2.19) and (3.3) that

$$
\frac{1}{2} \frac{d}{d t}\|f\|^{2}+\sigma\left\|\mathbf{P}_{1} f\right\|_{\nu}^{2}+\varepsilon\left\|\nabla_{v} f+\frac{v}{2} f\right\|^{2} \leq C \mid\|f\|\|\cdot\|\|f\|_{\nu}^{2} .
$$

Taking summation $d(3.5)+3$ (3.4) with $d>0$ a large constant and using (2.49), we have

$$
\begin{aligned}
& \frac{1}{2} \frac{d}{d t}\left\{d\|f\|^{2}+\sum_{\alpha>0}\left\|\partial^{\alpha} f\right\|^{2}-2 C_{1} \int_{\mathbb{R}^{3}} \nabla \cdot \mathbf{b} a d x\right\}+\sigma_{0}\left\{\left\|\mathbf{P}_{1} f\right\|_{\nu}^{2}+\sum_{\alpha>0}\left\|\partial^{\alpha} f\right\|_{\nu}^{2}\right\} \\
& \quad+\varepsilon \sum_{\alpha \geq 0}\left\|\nabla_{v} \partial^{\alpha} f+\frac{v}{2} \partial^{\alpha} f\right\|^{2} \leq C|\|f\|\|\cdot\||\|f\| \|_{\nu}^{2} .
\end{aligned}
$$

Next, we estimate the mixed derivative of $f$. Taking the inner product between $\partial_{\beta}^{\alpha}(1.5)$ and $\omega^{2|\beta|} \partial_{\beta}^{\alpha} f$ with $|\alpha|+|\beta| \leq N$, we get

$$
\begin{aligned}
& \frac{1}{2} \frac{d}{d t}\left\|\omega^{|\beta|} \partial_{\beta}^{\alpha} f\right\|^{2}+\left(C_{\beta}^{\beta_{1}} \partial_{\beta_{1}} v_{j} \partial_{x_{j}} \partial_{\beta-\beta_{1}}^{\alpha} f, \omega^{2|\beta|} \partial_{\beta}^{\alpha} f\right)+\left(\partial_{\beta}^{\alpha}(\mathbf{L} f), \omega^{2|\beta|} \partial_{\beta}^{\alpha} f\right) \\
& \quad-\varepsilon\left(\partial_{\beta}^{\alpha} \mathbf{F} f, \omega^{2|\beta|} \partial_{\beta}^{\alpha} f\right)=\left(\partial_{\beta}^{\alpha} \Gamma(f, f), \omega^{2|\beta|} \partial_{\beta}^{\alpha} f\right),
\end{aligned}
$$

where $\left|\beta_{1}\right|=1$. First we consider the case $\alpha>0$. For the second term on the left-hand side of (3.7),

$$
\begin{aligned}
\left(C_{\beta}^{\beta_{1}} \partial_{\beta_{1}} v_{j} \partial^{j} \partial_{\beta-\beta_{1}}^{\alpha} f, \omega^{2|\beta|} \partial_{\beta}^{\alpha} f\right) & \leq C\left\|\omega^{|\beta|-\frac{1}{2}} \partial_{\beta-1}^{\alpha+1} f\right\|\left\|\omega^{|\beta|+\frac{1}{2}} \partial_{\beta}^{\alpha} f\right\| \\
& \leq \eta\left\|\omega^{|\beta|} \partial_{\beta}^{\alpha} f\right\|_{\nu}^{2}+C_{\eta}\left\|\omega^{|\beta|-1} \partial_{\beta-1}^{\alpha+1} f\right\|_{\nu}^{2} .
\end{aligned}
$$

For the third term on the left-hand side, by (2.12), we have for any $\eta>0$ that

$$
\left(\omega^{2|\beta|} \partial_{\beta}^{\alpha}(\mathbf{L} f), \partial_{\beta}^{\alpha} f\right) \geq \frac{1}{2}\left\|\omega^{|\beta|} \partial_{\beta}^{\alpha} f\right\|_{\nu}^{2}-\eta \sum_{\beta_{1} \leq \beta}\left\|\omega^{\left|\beta_{1}\right|} \partial_{\beta_{1}}^{\alpha} f\right\|_{\nu}^{2}-C_{\eta}\left\|\partial^{\alpha} f\right\|_{\nu}^{2} .
$$

Therefore, by (2.18) and (2.50)-(2.52), we have

$$
\begin{aligned}
& \frac{d}{d t}\left\|\omega^{|\beta|} \partial_{\beta}^{\alpha} f\right\|^{2}+\left\|\omega^{|\beta|} \partial_{\beta}^{\alpha} f\right\|_{\nu}^{2}+\varepsilon\left\|\omega^{\beta} \nabla_{v} \partial_{\beta}^{\alpha} f\right\|^{2}+\frac{\varepsilon}{4}\left\|v \omega^{\beta} \partial_{\beta}^{\alpha} f\right\|^{2}+C \sqrt{\varepsilon}\left\|\omega^{|\beta|} \partial_{\beta}^{\alpha} f\right\|^{2} \\
& \leq C_{\eta}\left\|\omega^{|\beta|-1} \partial_{\beta-1}^{\alpha+1} f\right\|_{\nu}^{2}+C_{\eta}\left\|\partial^{\alpha} f\right\|_{\nu}^{2}+\eta \sum_{\beta_{1} \leq \beta}\left\|\omega^{\left|\beta_{1}\right|} \partial_{\beta_{1}}^{\alpha} f\right\|_{\nu}^{2}+C|\|f\|\|\cdot\||\|f\|_{\nu}^{2} \\
& \quad+C \varepsilon\left(\left\|\omega^{|\beta|-1} \partial_{\beta-1}^{\alpha} f\right\|^{2}+\left\|\omega^{|\beta|-2} \partial_{\beta-2}^{\alpha} f\right\|^{2}\right)
\end{aligned}
$$

For the case $\alpha=0$, we take $\mathbf{P}_{1}$ in (1.5) to get

$$
\partial_{t} \mathbf{P}_{1} f+v \cdot \nabla_{x} f-\mathbf{L} \mathbf{P}_{1} f-\varepsilon \mathbf{F} \mathbf{P}_{1} f=\mathbf{P}_{0}\left(v \cdot \nabla_{x} f\right)+\Gamma(f, f) .
$$

Taking the inner product between $\partial_{\beta}(3.11)$ and $\omega^{2|\beta|} \partial_{\beta} \mathbf{P}_{1} f$ over $L^{2}\left(\mathbb{R}_{x}^{3} \times \mathbb{R}_{v}^{3}\right)$, we get

$$
\begin{aligned}
& \frac{1}{2} \frac{d}{d t}\left\|\omega^{|\beta|} \partial_{\beta} \mathbf{P}_{1} f\right\|^{2}+\left(C_{\beta}^{\beta_{1}} \partial_{\beta_{1}} v_{j} \partial_{x_{j}} \partial_{\beta-\beta_{1}} f, \omega^{2|\beta|} \partial_{\beta} \mathbf{P}_{1} f\right)+\left(v \cdot \nabla_{x} \partial_{\beta} \mathbf{P}_{0} f, \omega^{2|\beta|} \partial_{\beta} \mathbf{P}_{1} f\right) \\
& \quad+\left(\partial_{\beta} \mathbf{L} \mathbf{P}_{1} f, \omega^{2|\beta|} \partial_{\beta} \mathbf{P}_{1} f\right)-\varepsilon\left(\partial_{\beta} \mathbf{F P}_{1} f, \omega^{2|\beta|} \partial_{\beta} \mathbf{P}_{1} f\right) \\
& =\left(\partial_{\beta} \mathbf{P}_{0}\left(v \cdot \nabla_{x} f\right), \omega^{2|\beta|} \partial_{\beta} \mathbf{P}_{1} f\right)+\left(\partial_{\beta} \Gamma(f, f), \omega^{2|\beta|} \partial_{\beta} \mathbf{P}_{1} f\right) .
\end{aligned}
$$

For the third term on the left-hand side and the first term on the right-hand side, it follows from (2.1) that

$$
\begin{aligned}
\left(v \cdot \nabla_{x} \partial_{\beta} \mathbf{P}_{0} f, \omega^{2|\beta|} \partial_{\beta} \mathbf{P}_{1} f\right) & =\left(v \cdot \nabla_{x} \partial_{\beta}\left\{\left(a+b_{j} v_{j}+c|v|^{2}\right) \sqrt{\mu}\right\}, \omega^{2|\beta|} \partial_{\beta} \mathbf{P}_{1} f\right) \\
& \leq \eta\left\|\omega^{|\beta|} \partial_{\beta} \mathbf{P}_{1} f\right\|_{\nu}^{2}+C_{\eta}\left\|\nabla_{x} \mathbf{P}_{0} f\right\|^{2},
\end{aligned}
$$




$$
\begin{aligned}
\left(\partial_{\beta} \mathbf{P}_{0}\left(v \cdot \nabla_{x} f\right), \omega^{2|\beta|} \partial_{\beta} \mathbf{P}_{1} f\right) & \leq C \sum_{i=0}^{4}\left|\left(v \cdot \nabla_{x} f, \psi_{i}\right)\left(\partial_{\beta} \psi_{i}, \omega^{2|\beta|} \partial_{\beta} \mathbf{P}_{1} f\right)\right| \\
& \leq \eta\left\|\omega^{|\beta|} \partial_{\beta} \mathbf{P}_{1} f\right\|_{\nu}^{2}+C_{\eta}\left\|\nabla_{x} f\right\|_{\nu}^{2}
\end{aligned}
$$

where $\left\{\psi_{i}, 0 \leq i \leq 4\right\}$ is defined by (2.53). Therefore, by (2.20), (3.13) and (3.14) we have

$$
\begin{aligned}
\frac{d}{d t}\left\|\omega^{|\beta|} \partial_{\beta} \mathbf{P}_{1} f\right\|^{2}+\left\|\omega^{|\beta|} \partial_{\beta} \mathbf{P}_{1} f\right\|_{\nu}^{2}+\varepsilon\left\|\omega^{|\beta|} \nabla_{v} \partial_{\beta} \mathbf{P}_{1} f\right\| \\
\quad+\frac{1}{4} \varepsilon\left\|v \omega^{|\beta|} \partial_{\beta} \mathbf{P}_{1} f\right\|^{2}+C \sqrt{\varepsilon}\left\|\omega^{|\beta|} \partial_{\beta} \mathbf{P}_{1} f\right\|^{2} \\
\leq C_{\eta}\left\|\omega^{|\beta|-1} \partial_{\beta-1}^{1} f\right\|_{\nu}^{2}+C_{\eta}\left(\left\|\nabla_{x} f\right\|_{\nu}^{2}+\left\|\nabla_{x} \mathbf{P}_{0} f\right\|^{2}\right)+C_{\eta}\left\|\mathbf{P}_{1} f\right\|_{\nu}^{2}+C\left|\left\|f \left|\|\cdot \mid\| f\|\|_{\nu}^{2}\right.\right.\right. \\
\quad+\eta \sum_{\beta_{1} \leq \beta}\left\|\omega^{\left|\beta_{1}\right|} \partial_{\beta_{1}} \mathbf{P}_{1} f\right\|_{\nu}^{2}+C \varepsilon\left(\left\|\omega^{|\beta|-1} \partial_{\beta-1} \mathbf{P}_{1} f\right\|^{2}+\left\|\omega^{|\beta|-2} \partial_{\beta-2} \mathbf{P}_{1} f\right\|^{2}\right) .
\end{aligned}
$$

Taking the summation $K(\underline{3.6})+\sum_{\alpha>0,|\alpha|+|\beta| \leq N} d_{\beta}^{\alpha}(\underline{(3.10)})+d_{0} \sum_{0<|\beta| \leq N}(\underline{3.15})$, where $K>0$ is a constant large enough, $\eta>0$ and $d_{0}>0$ are two constants small enough, and $d_{\beta}^{\alpha}>0$ is chosen properly such that

$$
d_{\beta-1}^{\alpha+1} / 2 \geq C_{\eta} d_{\beta}^{\alpha}, \quad|\alpha|+|\beta| \leq N
$$

we have

$$
\begin{aligned}
& \frac{d}{d t}\left\{K\left(d\|f\|^{2}+\sum_{\alpha>0}\left\|\partial^{\alpha} f\right\|^{2}-2 C_{1} \int_{\mathbb{R}^{3}} \nabla \cdot b a d x\right)+\sum_{\alpha>0}\left\|\omega^{|\beta|} \partial_{\beta}^{\alpha} f\right\|^{2}+\sum_{\beta>0}\left\|\omega^{|\beta|} \partial_{\beta} \mathbf{P}_{1} f\right\|^{2}\right\} \\
& \quad+\left\{K \sigma_{0}\left(\left\|\mathbf{P}_{1} f\right\|^{2}+\sum_{\alpha>0}\left\|\partial^{\alpha} f\right\|^{2}\right)+\sum_{\alpha>0}\left\|\omega^{|\beta|} \partial_{\beta}^{\alpha} f\right\|^{2}+\sum_{\beta>0}\left\|\omega^{|\beta|} \partial_{\beta} \mathbf{P}_{1} f\right\|^{2}\right\} \\
& \quad+\varepsilon \sum_{\alpha>0}\left(\left\|\omega^{|\beta|} \nabla_{v} \partial_{\beta}^{\alpha} f\right\|^{2}+\left\|v \mid \omega^{|\beta|} \partial_{\beta}^{\alpha} f\right\|^{2}\right) \leq C\left|\left\|f \left|\left\|\cdot \left|\|f \mid\|_{\nu}^{2}\right.\right.\right.\right.\right.
\end{aligned}
$$

which implies

$$
\begin{aligned}
& \|\| f(t) \mid \|^{2}+\int_{0}^{t}\left(\sigma _ { 0 } \left|\left\|f(s)||_{\nu}^{2}+\left.\varepsilon|\| f(s)|\right|_{F P} ^{2}\right) d s\right.\right. \\
& \leq C\left|\left\|f_{0}\right\|\right|^{2}+C \sup _{0 \leq s \leq t}\left|\left\|f ( s ) \left|\left\|\int _ { 0 } ^ { t } \left|\|f(s) \mid\|_{\nu}^{2} d s .\right.\right.\right.\right.\right.
\end{aligned}
$$

We take $\delta_{1}>0$ small such that $\sigma_{0}-C \delta_{1} \geq \sigma_{0} / 2$. Then

$$
\left.\||| f(t)\|\right|^{2}+\int_{0}^{t}\left(\left|\left\|f(s)\left|\left\|_{\nu}^{2}+\varepsilon|\|f(s)\||_{F P}^{2}\right) d s \leq C\right|\right\| f_{0} \|\right|^{2} .\right.
$$

Therefore, the small assumption (3.2) can be verified so long as $\delta>0$ is small enough so that $C \delta \leq \delta_{1}$. With the help of uniformly a priori estimates (3.18) and the short time existence of the unique classical solution to IVP (1.5)-(1.6), we can extend the short time classical solution globally in time by the standard continuity argument. The proof is completed. 


\section{Long time behavior}

Let $\|\cdot\|_{H^{k}}$ be the norm in the space $H^{k}\left(\mathbb{R}_{x}^{3}\right) \times L^{2}\left(\mathbb{R}_{v}^{3}\right)$, which is defined by

$$
\|f\|_{H^{k}}=\left(\int_{\mathbb{R}_{v}^{3}} \int_{\mathbb{R}_{\xi}^{3}}\left(1+|\xi|^{2}\right)^{k}|\hat{f}(\xi, v)|^{2} d \xi d v\right)^{\frac{1}{2}},
$$

where $\hat{f}$ denotes the Fourier transform of $f$. As in [20, 11], we introduce the compensating function below.

Definition 4.1 (Compensating function). Let $S(\omega), \omega \in \mathbb{S}^{2}$, be a bounded linear operator on $L^{2}\left(\mathbb{R}_{v}^{3}\right)$. Here $S$ is called a compensating function for the Boltzmann equation if

(i) $S(\omega)$ is $C^{\infty}$ on $\mathbb{S}^{2}$ with values in the space of bounded linear operators on $L^{2}\left(\mathbb{R}_{v}^{3}\right)$ and $S(-\omega)=-S(\omega)$ for $\omega \in \mathbb{S}^{2}$,

(ii) $i S(\omega)$ is self-adjoint on $L^{2}\left(\mathbb{R}_{v}^{3}\right)$, for all $\omega \in \mathbb{S}^{2}$,

(iii) there exists $\delta_{0}$ such that

$$
\mathcal{R}\langle S(\omega)(\omega \cdot v) f, f\rangle-\langle\mathbf{L} f, f\rangle \geq \delta_{0}|f|_{\nu}^{2}, \quad \text { for all } f \in L^{2}\left(\mathbb{R}_{v}^{3}\right), \omega \in \mathbb{S}^{2} .
$$

Let $W$ be the linear space spanned by (2.4), and let $\left\{e_{i}, 1 \leq i \leq 13\right\}$ be the normalized orthogonal basis of $W$. Given $\omega \in \mathbb{S}^{2}$, let

$$
S(\omega) f=\sum_{k, l=1}^{13} \lambda r_{k l}(\omega)\left\langle f, e_{l}\right\rangle e_{k}, \quad \beta>0, f \in L^{2}\left(\mathbb{R}_{v}^{3}\right) .
$$

Then, making use of arguments similar to those proving Lemma 3.11.2 in [11, we obtain the following result.

Lemma 4.2. There exists $\lambda>0$ such that $S(\omega)$ defined by (4.3) is a compensating function of the FPB equation

$$
f_{t}+v \cdot \nabla_{x} f-\mathbf{L} f-\varepsilon \mathbf{F} f=\Gamma(f, f) .
$$

Moreover, $S(\omega): L^{2}\left(\mathbb{R}_{v}^{3}\right) \rightarrow W$.

With the help of Lemma 4.2. we can obtain the time-decay rates of the solution to the IVP (1.5)- (1.6).

Lemma 4.3. Let $f$ be the solution of the IVP (1.5)-(1.6). Then for sufficiently small $\varepsilon>0$

$$
\begin{aligned}
& \|f(t)\|_{H^{k}}^{2} \leq C(1+\sqrt{\varepsilon} t)^{-\frac{3}{2}}\left(\left\|f_{0}\right\|_{Z_{1}}^{2}+\left\|f_{0}\right\|_{H^{k}}^{2}\right) \\
& \quad+C \int_{0}^{t}(1+\sqrt{\varepsilon}(t-s))^{-\frac{3}{2}}\left(\left\|\nu^{-\frac{1}{2}} \Gamma(f(s))\right\|_{Z_{1}}^{2}+\left\|\nu^{-\frac{1}{2}} \Gamma(f(s))\right\|_{H^{k}}^{2}\right) d s,
\end{aligned}
$$

for any integer $k \geq 0$.

Proof. Let $\omega=\frac{\xi}{|\xi|}$ and take the Fourier transform of (1.5) in $x$ :

$$
\hat{f}_{t}+i|\xi|(v \cdot \omega) \hat{f}-\mathbf{L} \hat{f}-\varepsilon \mathbf{F} \hat{f}=\hat{\Gamma}, \quad \hat{f}(\xi, v, 0)=\hat{f}_{0}(\xi, v) .
$$


Let $S(\omega)$ be a compensating function defined by (4.3), and let $\kappa>0$ be a small constant. Set

$$
E(\hat{f})(t, \xi)=|\hat{f}(t, \xi, \cdot)|_{2}^{2}-\frac{\kappa|\xi|}{1+|\xi|^{2}}\langle i S(\omega) \hat{f}(t, \xi, \cdot), \hat{f}(t, \xi, \cdot)\rangle,
$$

where $\langle\cdot, \cdot\rangle$ and $|\cdot|_{2}$ denote the inner product and norm over $L^{2}\left(\mathbb{R}_{v}^{3}\right)$. It is easy to verify for small $\kappa>0$ that

$$
\frac{1}{2}|\hat{f}|_{2}^{2} \leq E(\hat{f}) \leq 2|\hat{f}|_{2}^{2}
$$

Taking the inner product between (4.5) and $\hat{f}$ and choosing the real part, we get

$$
\frac{1}{2} \frac{d}{d t}|\hat{f}|_{2}^{2}-\langle\mathbf{L} \hat{f}, \hat{f}\rangle-\varepsilon\langle\mathbf{F} \hat{f}, \hat{f}\rangle=\mathcal{R}\langle\hat{\Gamma}, \hat{f}\rangle .
$$

Next apply $-i|\xi| S(\omega)$ to (4.5). We have

$$
-i|\xi| S(\omega) \hat{f}_{t}+|\xi|^{2} S(\omega)((v \cdot \omega) \hat{f})+i|\xi| S(\omega) \mathbf{L} \hat{f}+\varepsilon i|\xi| S(\omega) \mathbf{F} \hat{f}=-i|\xi| S(\omega) \hat{\Gamma} .
$$

Taking the inner product between the above and $\hat{f}$ and choosing the real part, it follows that

$$
\begin{aligned}
& \mathcal{R}\left\langle-i|\xi| S(\omega) \hat{f}_{t}, \hat{f}\right\rangle+|\xi|^{2} \mathcal{R}\langle S(\omega)(v \cdot \omega) \hat{f}, \hat{f}\rangle \\
& =-|\xi|\{\mathcal{R}\langle i S(\omega) \mathbf{L} \hat{f}, \hat{f}\rangle+\varepsilon \mathcal{R}\langle i S(\omega) \mathbf{F} \hat{f}, \hat{f}\rangle+\mathcal{R}\langle i S(\omega) \hat{\Gamma}, \hat{f}\rangle\} .
\end{aligned}
$$

Taking the summation $\left(1+|\xi|^{2}\right)$ (4.7) $+\kappa(4.8)$, we have

$$
\begin{aligned}
& \frac{d}{d t}\left\{\frac{(1+|\xi|)^{2}}{2} E(\hat{f})\right\}-\left(1+(1-\kappa)|\xi|^{2}\right)\langle\mathbf{L} \hat{f}, \hat{f}\rangle \\
& \quad+\kappa|\xi|^{2}\{\mathcal{R}\langle S(\omega)(v \cdot \omega) \hat{f}, \hat{f}\rangle+\langle\mathbf{L} \hat{f}, \hat{f}\rangle\}-\varepsilon\left(1+|\xi|^{2}\right)\langle\mathbf{F} \hat{f}, \hat{f}\rangle \\
& =-\kappa|\xi|\{\mathcal{R}\langle i S(\omega) \mathbf{L} \hat{f}, \hat{f}\rangle+\varepsilon \mathcal{R}\langle i S(\omega) \mathbf{F} \hat{f}, \hat{f}\rangle+\mathcal{R}\langle i S(\omega) \hat{\Gamma}, \hat{f}\rangle\}+\left(1+|\xi|^{2}\right) \mathcal{R}\langle\hat{\Gamma}, \hat{f}\rangle,
\end{aligned}
$$

where

$$
\begin{aligned}
-\langle\mathbf{L} \hat{f}, \hat{f}\rangle & \geq \sigma\left|\mathbf{P}_{1} \hat{f}\right|_{\nu}^{2}, \\
S(\omega) \mathbf{L} \hat{f} & =\sum_{k, l} \lambda r_{k l}(\omega)\left\langle\mathbf{P}_{1} \hat{f}, \mathbf{L}\left(e_{l}\right)\right\rangle e_{k}, \\
S(\omega) \mathbf{F} \hat{f} & =\sum_{k, l} \lambda r_{k l}(\omega)\left\langle\mathbf{F} \hat{f}, e_{l}\right\rangle e_{k}=\sum_{k, l} \lambda r_{k l}(\omega)\left\langle\nabla_{v} \hat{f}+\frac{v}{2} \hat{f}, \sqrt{\mu} \nabla_{v}\left(e_{l} \mu^{-\frac{1}{2}}\right)\right\rangle e_{k} .
\end{aligned}
$$

Due to the facts that $\mathbf{P}_{0} \hat{\Gamma}=0, \mathbf{P}_{1} \hat{\Gamma}=\hat{\Gamma}$, it follows that

$$
\begin{aligned}
|\langle\hat{\Gamma}, \hat{f}\rangle| & =\left|\left\langle\hat{\Gamma}, \mathbf{P}_{1} \hat{f}\right\rangle\right| \leq\left|\nu^{-\frac{1}{2}} \hat{\Gamma}\right|_{2}\left|\mathbf{P}_{1} \hat{f}\right|_{\nu}, \\
|\langle S(\omega) \hat{\Gamma}, \hat{f}\rangle| & =\left|\sum_{k, l} \lambda r_{k l}(\omega)\left\langle\hat{\Gamma}, \mathbf{P}_{1} e_{l}\right\rangle\left\langle e_{k}, \hat{f}\right\rangle\right| \leq C\left|\nu^{-\frac{1}{2}} \hat{\Gamma}\right|_{2}\left|\mathbf{P}_{1} \hat{f}\right|_{\nu} .
\end{aligned}
$$

Thus, we have

$$
\begin{aligned}
& \kappa|\xi|[|\langle S(\omega) \mathbf{L} \hat{f}, \hat{f}\rangle|+|\varepsilon\langle i S(\omega) \mathbf{F} \hat{f}, \hat{f}\rangle|+|\langle S(\omega) \hat{\Gamma}, \hat{f}\rangle|] \\
& \leq c \kappa|\xi|\left[\left|\mathbf{P}_{1} \hat{f}\right|_{\nu}|\hat{f}|_{\nu}+\varepsilon\left|\nabla_{v} \hat{f}+\frac{v}{2} \hat{f}\right|_{2}|\hat{f}|_{\nu}+\left|\nu^{-\frac{1}{2}} \hat{\Gamma}\right|_{2}\left|\mathbf{P}_{1} \hat{f}\right|_{\nu}\right]
\end{aligned}
$$




$$
\leq \frac{\kappa \delta_{0}}{2}|\xi|^{2}|\hat{f}|_{\nu}^{2}+\frac{\kappa c^{2}}{\delta_{0}}\left|\mathbf{P}_{1} \hat{f}\right|_{\nu}^{2}+\frac{\kappa c^{2} \varepsilon^{2}}{\delta_{0}}\left|\nabla_{v} \hat{f}+\frac{v}{2} \hat{f}\right|_{2}^{2}+\frac{\kappa \sigma}{8}|\xi|^{2}\left|\mathbf{P}_{1} \hat{f}\right|_{\nu}^{2}+\frac{2 \kappa}{\sigma}\left|\nu^{-\frac{1}{2}} \hat{\Gamma}\right|_{2}^{2}
$$

and

$$
\left(1+|\xi|^{2}\right) \mathcal{R}\langle\hat{\Gamma}, \hat{f}\rangle \leq \frac{\sigma}{8}\left(1+|\xi|^{2}\right)\left|\mathbf{P}_{1} \hat{f}\right|_{\nu}^{2}+\frac{2}{\sigma}\left(1+|\xi|^{2}\right)\left|\nu^{-\frac{1}{2}} \hat{\Gamma}\right|_{2}^{2} .
$$

Choosing $\kappa>0$ small enough such that $\kappa<\min \left\{\frac{\sigma \delta_{0}}{c^{2}+\delta_{0}}, \frac{\delta_{0}}{\varepsilon c^{2}}, \frac{\sigma}{8}, \frac{\delta_{0} \sigma}{4 c^{2}}\right\}$, we have

$$
\begin{aligned}
& \frac{d}{d t}\left(1+|\xi|^{2}\right) E(\hat{f})+\kappa \delta_{0}|\xi|^{2}|\hat{f}|_{\nu}^{2} \\
& \quad+\frac{\sigma}{4}\left(1+|\xi|^{2}\right)\left|\mathbf{P}_{1} \hat{f}\right|_{\nu}^{2}-\varepsilon\left(1+|\xi|^{2}\right)\langle\mathbf{F} \hat{f}, \hat{f}\rangle \leq \frac{8}{\sigma}\left(1+|\xi|^{2}\right)\left|\nu^{-\frac{1}{2}} \hat{\Gamma}\right|_{2}^{2} .
\end{aligned}
$$

Therefore, if $\varepsilon>0$ is small, by (2.50), there exists a constant $\sigma_{1}>0$ such that

$$
\frac{d}{d t} E(\hat{f})+\sigma_{1} \sqrt{\varepsilon} \hat{\rho}(\xi) E(\hat{f}) \leq C\left|\nu^{-\frac{1}{2}} \hat{\Gamma}\right|_{2}^{2},
$$

where

By the Gronwall inequality, we have

$$
\hat{\rho}(\xi)=\frac{|\xi|^{2}}{1+|\xi|^{2}}
$$

$$
E(\hat{f}) \leq e^{-\sigma_{1} \sqrt{\varepsilon} t \hat{\rho}(\xi)} E\left(\hat{f}_{0}\right)+C \int_{0}^{t} e^{-\sigma_{1} \sqrt{\varepsilon}(t-s) \hat{\rho}(\xi)}\left|\nu^{-\frac{1}{2}} \hat{\Gamma}(s, \xi)\right|_{2}^{2} d s .
$$

Therefore by the inequality (4.6),

$$
|\hat{f}(t, \xi)|_{2}^{2} \leq C e^{-\sigma_{1} \sqrt{\varepsilon} t} \hat{\rho}(\xi)\left|\hat{f}_{0}(t, \xi)\right|_{2}^{2}+C \int_{0}^{t} e^{-\sigma_{1} \sqrt{\varepsilon}(t-s) \hat{\rho}(\xi)}\left|\nu^{-\frac{1}{2}} \hat{\Gamma}(s, \xi)\right|_{2}^{2} d s .
$$

Multiplying (4.13) with $\left(1+|\xi|^{2}\right)^{k}$ and integrating the resulting inequality with respect to $\xi$, we have

$$
\begin{aligned}
\|f(t)\|_{H^{k}}^{2}= & \int_{\mathbb{R}^{3}}\left(1+|\xi|^{2}\right)^{k}|\hat{f}(t, \xi)|_{2}^{2} d \xi \\
\leq & C \int_{\mathbb{R}^{3}}\left(1+|\xi|^{2}\right)^{k} e^{-\sigma_{1} \sqrt{\varepsilon} \frac{|\xi|^{2}}{1+|\xi|^{2}}}\left|\hat{f}_{0}(\xi)\right|_{2}^{2} d \xi \\
& +C \int_{0}^{t} \int_{\mathbb{R}^{3}}\left(1+|\xi|^{2}\right)^{k} e^{-\sigma_{1} \sqrt{\varepsilon}(t-s) \hat{\rho}(\xi)}\left|\nu^{-\frac{1}{2}} \hat{\Gamma}(s, \xi)\right|_{2}^{2} d \xi d s .
\end{aligned}
$$

The right-hand side terms can be estimated as follows:

$$
\begin{aligned}
I_{0} & =: \int_{\mathbb{R}^{3}}\left(1+|\xi|^{2}\right)^{k} e^{-\sigma_{1} \sqrt{\varepsilon} t \frac{|\xi|^{2}}{1+|\xi|^{2}}}\left|\hat{f}_{0}(\xi)\right|_{2}^{2} d \xi=\int_{|\xi|<\eta}+\int_{|\xi|>\eta} \\
& \leq \sup _{|\xi|<\eta}\left\|\hat{f}_{0}(\xi, \cdot)\right\|^{2}(1+\eta)^{k} \int_{|\xi|<\eta} e^{-\sigma_{1} \sqrt{\varepsilon} t \frac{|\xi|^{2}}{1+|\xi|^{2}}} d \xi+e^{-\sigma_{1} \sqrt{\varepsilon} t \frac{\eta^{2}}{1+\eta^{2}}\left\|f_{0}\right\|_{H^{k}}^{2}} \\
& \leq C(1+\eta)^{k}\left\|f_{0}\right\|_{Z_{1}}^{2}\left(\sigma_{1} \sqrt{\varepsilon} t\right)^{-\frac{3}{2}}\left(1+\eta^{2}\right)^{\frac{3}{2}}+e^{-\sigma_{1} \sqrt{\varepsilon} t \frac{\eta^{2}}{1+\eta^{2}}}\left\|f_{0}\right\|_{H^{k}}^{2} .
\end{aligned}
$$

Now we take $\eta=1$ and $t \geq 1$ (without loss of generality) to get

$$
\begin{aligned}
I_{0} & \leq e^{-\frac{\sigma_{1} \sqrt{\varepsilon} t}{2}}\left\|f_{0}\right\|_{H^{k}}^{2}+C(1+\sqrt{\varepsilon} t)^{-\frac{3}{2}}\left\|f_{0}\right\|_{Z_{1}}^{2} \\
& \leq C(1+\sqrt{\varepsilon} t)^{-\frac{3}{2}}\left(\left\|f_{0}\right\|_{H^{k}}^{2}+\left\|f_{0}\right\|_{Z_{1}}^{2}\right) .
\end{aligned}
$$


Similarly, we can show that

$$
\begin{aligned}
& \int_{0}^{t} \int_{\mathbb{R}^{3}}\left(1+|\xi|^{2}\right)^{k} e^{-\sigma_{1}(t-s) \hat{\rho}(\xi)}\left|\nu^{-\frac{1}{2}} \hat{\Gamma}(s, \xi)\right|_{2}^{2} d \xi d s \\
& \quad \leq C \int_{0}^{t}(1+\sqrt{\varepsilon}(t-s))^{-\frac{3}{2}}\left(\left\|\nu^{-\frac{1}{2}} \Gamma(f(s))\right\|_{Z_{1}}^{2}+\left\|\nu^{-\frac{1}{2}} \Gamma(f(s))\right\|_{H^{k}}^{2}\right) d s .
\end{aligned}
$$

Substituting (4.16)-(4.17) into (4.14), we obtain the expected estimates (4.4).

Lemma 4.4. Let $f$ be the solution of IVP (1.5)-11.6). There exist small constants $\delta>0, \varepsilon_{0}>0$ such that if $\left\|\left|f_{0} \|\right| \leq \delta\right.$ and $\varepsilon \in\left(0, \varepsilon_{0}\right]$, then

$$
\|f(t)\|_{H^{k}} \leq C\left(\left\|f_{0}\right\|_{H^{k}}+\left\|f_{0}\right\|_{Z_{1}}\right)(1+\sqrt{\varepsilon} t)^{-\frac{3}{4}}, \quad t>0 .
$$

Proof. From (4.4), we have

$$
\begin{aligned}
\|f\|_{H^{k}}^{2} \leq & C\left(\left\|f_{0}\right\|_{H^{k}}^{2}+\left\|f_{0}\right\|_{Z_{1}}^{2}\right)(1+\sqrt{\varepsilon} t)^{-\frac{3}{2}} \\
& +C \int_{0}^{t}(1+\sqrt{\varepsilon}(t-s))^{-\frac{3}{2}}\left(\left\|\nu^{-\frac{1}{2}} \Gamma(f)\right\|_{Z_{1}}^{2}+\left\|\nu^{-\frac{1}{2}} \Gamma(f)\right\|_{H^{k}}^{2}\right) d s .
\end{aligned}
$$

From (2.37), (2.38) and (3.18), the term $\Gamma(f, f)$ satisfies

$$
\begin{aligned}
\left\|\nu^{-\frac{1}{2}} \Gamma(f, f)\right\|_{Z_{1}}^{2} & \leq C \sum_{|\beta| \leq 2}\left\|\omega^{|\beta|} \partial_{\beta} f\right\|^{2} \times\|f\|_{\nu}^{2} \leq C \delta^{2}\|f\|^{2}, \\
\left\|\nu^{-\frac{1}{2}} \Gamma(f, f)\right\|_{H^{k}}^{2} & \leq C \sum_{\left|\alpha_{1}\right|+\left|\alpha_{2}\right| \leq N}\left\|\nu^{-\frac{1}{2}} \Gamma\left(\partial^{\alpha_{1}} f, \partial^{\alpha_{2}} f\right)\right\|^{2} \\
& \leq C \sum_{|\alpha|+|\beta| \leq N}\left\|\omega^{|\beta|} \partial_{\beta}^{\alpha} f\right\|^{2} \sum_{\left|\alpha_{2}\right| \leq N}\left\|\partial^{\alpha_{2}} f\right\|_{\nu}^{2} \leq C \delta^{2}\|f\|_{H^{k}}^{2} .
\end{aligned}
$$

Therefore,

$$
\|f\|_{H^{k}}^{2} \leq C\left(\left\|f_{0}\right\|_{H^{k}}^{2}+\left\|f_{0}\right\|_{Z_{1}}^{2}\right)(1+\sqrt{\varepsilon} t)^{-\frac{3}{2}}+C \delta^{2} \int_{0}^{t}(1+\sqrt{\varepsilon}(t-s))^{-\frac{3}{2}}\|f(s)\|_{H^{k}}^{2} d s .
$$

Define

$$
Q(t)=: \sup _{0 \leq s \leq t}\left\{(1+\sqrt{\varepsilon} s)^{-\frac{3}{2}}\|f(s)\|_{H^{k}}^{2}\right\}
$$

It follows that

$$
\begin{aligned}
\|f\|_{H^{k}}^{2} \leq & C\left(\left\|f_{0}\right\|_{H^{k}}^{2}+\left\|f_{0}\right\|_{Z_{1}}^{2}\right)(1+\sqrt{\varepsilon} t)^{-\frac{3}{2}} \\
& +C \delta^{2} \int_{0}^{t}(1+\sqrt{\varepsilon}(t-s))^{-\frac{3}{2}}(1+\sqrt{\varepsilon} s)^{-\frac{3}{2}} d s Q(t) \\
\leq & C(1+\sqrt{\varepsilon} t)^{-\frac{3}{2}}\left(\left\|f_{0}\right\|_{H^{k}}^{2}+\left\|f_{0}\right\|_{Z_{1}}^{2}+\delta^{2} Q(t)\right)
\end{aligned}
$$

i.e.,

$$
Q(t) \leq C\left(\left\|f_{0}\right\|_{H^{k}}^{2}+\left\|f_{0}\right\|_{Z_{1}}^{2}+\delta^{2} Q(t)\right) .
$$

Therefore if $\delta>0$ is small enough, we have

$$
Q(t) \leq C\left(\left\|f_{0}\right\|_{H^{k}}^{2}+\left\|f_{0}\right\|_{Z_{1}}^{2}\right),
$$

which implies (4.18), and the proof is completed. 
Proof of long time behavior in Theorem 1.1. We add $\|f\|^{2}$ to both sides of (3.16) to get

where

$$
\frac{d}{d t} E(t)+D(t) \leq C\|f(t)\|^{2}
$$

$$
\begin{aligned}
& E(t)=K\left(d\|f\|^{2}+\sum_{\alpha>0}\left\|\partial^{\alpha} f\right\|^{2}-2 C_{1} \int_{\mathbb{R}^{3}} \nabla \cdot b a d x\right)+\sum_{|\alpha|+|\beta| \leq N}\left\|\omega^{|\beta|} \partial_{\beta}^{\alpha} f\right\|^{2}, \\
& D(t)=\sigma_{0} \sum_{|\alpha|+|\beta| \leq N}\left\|\omega^{|\beta|} \partial_{\beta}^{\alpha} f\right\|_{\nu}^{2}+\varepsilon \sum_{|\alpha|+|\beta| \leq N}\left(\left\|\omega^{|\beta|} \nabla_{v} \partial_{\beta}^{\alpha} f\right\|^{2}+\left\|v \omega^{|\beta|} \partial_{\beta}^{\alpha} f\right\|^{2}\right) .
\end{aligned}
$$

Since

$$
\sigma_{0}\left\|\omega^{|\beta|} \partial_{\beta}^{\alpha} f\right\|_{\nu}^{2}+\varepsilon\left\|v \omega^{|\beta|} \partial_{\beta}^{\alpha} f\right\|^{2} \geq C_{0} \sqrt{\varepsilon}\left\|\omega^{|\beta|} \partial_{\beta}^{\alpha} f\right\|^{2},
$$

we have by (4.18) that for any $t>0$

$$
\frac{d}{d t} E(t)+C_{0} \sqrt{\varepsilon} E(t) \leq C\|f(t)\|^{2} \leq C\left(\left\|f_{0}\right\|_{H^{k}}+\left\|f_{0}\right\|_{Z_{1}}\right)^{2}(1+\sqrt{\varepsilon} t)^{-\frac{3}{2}} .
$$

Applying the Gronwall inequality to (4.22) concludes that

$$
\begin{aligned}
E(t) & \leq e^{-C_{0} \sqrt{\varepsilon} t} E(0)+C \int_{0}^{t} e^{-C_{0} \sqrt{\varepsilon}(t-s)}\|f(s)\|^{2} d s \\
& \leq e^{-C_{0} \sqrt{\varepsilon} t} E(0)+C \int_{0}^{t} e^{-C_{0} \sqrt{\varepsilon}(t-s)}(1+\sqrt{\varepsilon} s)^{-\frac{3}{2}} d s\left(\left\|f_{0}\right\|_{H^{k}}+\left\|f_{0}\right\|_{Z_{1}}\right)^{2} \\
& \leq C(1+\sqrt{\varepsilon} t)^{-\frac{3}{2}}\left[E(0)+\left(\left\|f_{0}\right\|_{H^{k}}+\left\|f_{0}\right\|_{Z_{1}}\right)^{2}\right] .
\end{aligned}
$$

By (4.23) and the fact that

$$
C_{1}\left|\| f ( t ) \| \left\|^{2} \leq E(t) \leq C_{2}\left|\|f(t) \mid\|^{2},\right.\right.\right.
$$

we obtain (1.12). The proof is completed.

\section{Acknowledgments}

The authors would like to thank the referee for his helpful suggestions on the paper. They also would like to thank Professor Fucai Li for the helpful comments on the paper.

\section{REFERENCES}

[1] Arnold, A., Markowich, P.A., Toscani, G., Unterreiter, A., On convex Sobolev inequalities and the rate of convergence to equilibrium for Fokker-Planck type equations. Comm Partial Differential Equations 26 (2001), 43-100. MR1842428(2002d:35097)

[2] Bisi, M., Carrillo, J.A., Toscani, G., Contractive metrics for a Boltzmann equation for granular gases: diffusive equilibria. J. Stat. Phys. 118 (2005), 301-331. MR 2122557 (2006d:82061)

[3] Carrillo, J.A., Toscani, G., Exponential convergence toward equilibrium for homogeneous FokkerPlanck-type equations. Math. Methods Appl. Sciences 21 (1998), 1269-1286. MR1639292 (99h:82055)

[4] Cerergnani, C., The Boltzmann equation and Its Application. Springer-Verlag, 1988. MR1313028 (95i:82082)

[5] Caflisch, Russel E., The Boltzmann equation with a soft potential, I. Linear, spatially-homogeneous. Comm. Math. Phys. 74 (1980), 71-95. MR575897(82j:82040a)

[6] Caflisch, Russel E., The Boltzmann equation with a soft potential, II. Nonlinear, spatially-periodic. Comm. Math. Phys. 74 (1980), 97-107. MR576265(82j:82040b) 
[7] Desvillettes, L., Villani, C., On the trend to global equilibrium in spatially inhomogeneous entropydissipating systems. Part I: The linear Fokker-Planck equation. Comm. Pure Appl. Math. 54 (2001), 1-42. MR.1787105 (2001h:82079)

[8] Desvillettes, L., Villani, C., On the trend to global equilibrium for spatially inhomogeneous kinetic systems: The Boltzmann equation. Invent. Math. 159 (2005), 245-316. MR2116276 (2005j:82070)

[9] DiPerna, R.J., Lion, P.L., On the Cauchy problem for Boltzmann equations: global existence and weak stability. Ann. Math. 130 (1989), 321-366. MR1014927 (90k:82045)

[10] DiPerna, R.J., Lion, P.L., On the Fokker-Planck-Boltzmann equation. Comm. Math. Phys. 120 (1988), 1-23. MR.972541 (90b:35203)

[11] Glassey, R.T., The Cauchy Problem in Kinetic Theory. SIAM, Philadelphia, PA, 1996. MR1379589 (97i:82070)

[12] Grad, H., Asymptotic theory of the Boltzmann equation. II. Rarefied Gas Dynamics. Ed. J.A. Laurmann, Vol. I, 26-59, Academic Press, New York, 1963. MR0156656 (27:6577)

[13] Guo, Y., The Boltzmann equation in the whole space, Indiana Univ. Math. J. 53 (2004), 1081-1094. MR 2095473 (2005g:35028)

[14] Guo, Y., The Vlasov-Poisson-Boltzmann system near Maxwellians, Comm. Pure. Appl. Math. 55(9) (2002), 1104-1135. MR1908664 (2003b:82050)

[15] Guo, Y., The Vlasov-Maxwell-Boltzmann system near Maxwellians, Invent. Math. 153(3) (2003), 593-630. MR2000470 (2004m:82123)

[16] Guo, Y., Classical Solutions to the Boltzmann Equation for Molecules with an Angular Cutoff, Arch. Ration. Mech. Anal. 169 (2003), 305-353. MR2013332 (2004i:82054)

[17] Hamdache, K., Estimations uniformes des solutions de l'équation de Boltzmann par les méthodes de viscosité artificielle et de diffusion de Fokker Planck. C. R. Math. Acad. Sci. Paris 302 (1986), 187-190. MR 832069 (88d:82060)

[18] Hamdache, K., Théorémes de compacité par compensation et applications en théorie cintique des gaz [Compensated compactness theorems and applications to the kinetic theory of gases]. C. $R$. Math. Acad. Sci. Paris 302 (1986), 151-154. MR832059(88d:82059)

[19] Hsiao, L., Yu, H.J., On the Cauchy Problem of the Boltzmann and Landau Equation with soft potentials, Quarterly of Applied Math. Volume LXV, Number 2 (2007), 281-315. MR2330559 (2008i:35235)

[20] Kawashima, S., The Boltzmann equation and thirteen moments. Japan J. Appl. Math. 7 (1990), no. 2, 301-320. MR1057534(91i:82020)

[21] Li, H.L., Matsumura, A., Behaviour of the Fokker-Planck-Boltzmann equation near a Maxwellian, Arch. Ration. Mech. Anal. 189 (2008), 1-44. MR2403598 (2009d:82117)

[22] Li, H.L, Diffusive Property of the Fokker-Planck-Boltzmann Equation, Reprint from Bulletin of the Institute of Mathematics, Academia Sinica (New Series), Volume 2, No. 4, pp. 921-933, December 2007. MR 2363984 (2009b:82087)

[23] Liu, T.P., Yang, T., Yu, S.H., Energy method for Boltzmann equation. Physica D 188 (2004), 178-192. MR 2043729(2005a:82091)

[24] Liu, T.P., Yu, S.H., Boltzmann equation: Micro-macro decompositions and positivity of shock profiles. Comm. Math. Phys. 246 (2004), No. 1, 133-179. MR2044894 (2005f:82101)

[25] Liu, T.P., Yang, T., Yu, S.H., Zhao, H.J., Nonlinear Stability of Rarefaction Waves for the Boltzmann Equation. Arch. Ration. Mech. Anal. 181 (2006), 333-371. MR2221210(2007b:76106)

[26] Loyalka, S.K., Rarefied gas dynamic problems in enviromental sciences. Proceedings 15th International Symposum on Rarefied Gas Dynamics, held in Grado, June 16-20 (Eds. V. Boffi and C. Cercignani), Teubner, Stuttgart, 1986.

[27] Toscani, G., Entropy production and the rate of convergence to equilibrium for the Fokker-Planck equation. Quart. Appl. Math. 57 (1999), no. 3, 521-541. MR1704435(2000m:82042)

[28] Strain, R.M., Guo, Y., Exponential decay for soft potentials near Maxwellian. Arch. Ration. Mech. Anal. 187 (2008), no. 2, 287-339. MR2366140(2008m:82008)

[29] Strain, R.M., Optimal time decay of the non-cut-off Boltzmann equation in the whole space, arXiv:1011.5561v2

[30] Ukai, S., Asano, K., On the Cauchy problem of the Boltzmann equation with a soft potential, Publ. Res. Inst. Math. Sci. 18 (1982), 477-519. MR677262(84h:82048)

[31] Yang, T., Yu, H., Hypocoercivity of the relativistic Boltzmann and Landau equations in the whole space, J. Differential Equations 248 (2010), 1518-1560. MR 2593052 
[32] Zhong, M.Y., Li, H.L., Long time behavior of the Fokker-Planck-Boltzmann equation, preprint, 2009 . 